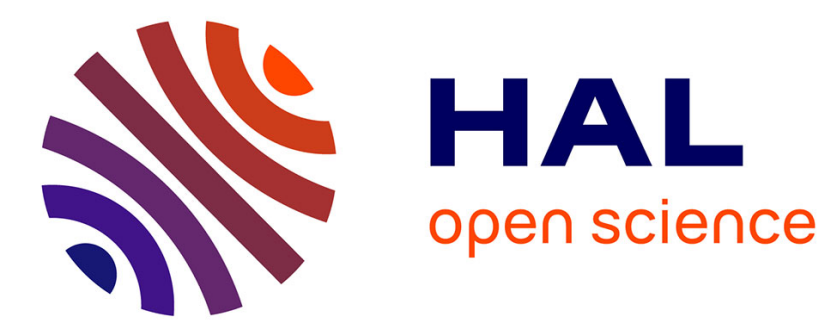

\title{
Sensitivity analysis for the outages of nuclear power plants
}

Kengy Barty, J. Frederic Bonnans, Laurent Pfeiffer

\section{To cite this version:}

Kengy Barty, J. Frederic Bonnans, Laurent Pfeiffer. Sensitivity analysis for the outages of nuclear power plants. Energy Systems, 2014, 5 (2), pp.371-406. 10.1007/s12667-013-0096-y · hal-00671186

\section{HAL Id: hal-00671186 \\ https://hal.inria.fr/hal-00671186}

Submitted on 16 Feb 2012

HAL is a multi-disciplinary open access archive for the deposit and dissemination of scientific research documents, whether they are published or not. The documents may come from teaching and research institutions in France or abroad, or from public or private research centers.
L'archive ouverte pluridisciplinaire HAL, est destinée au dépôt et à la diffusion de documents scientifiques de niveau recherche, publiés ou non, émanant des établissements d'enseignement et de recherche français ou étrangers, des laboratoires publics ou privés. 


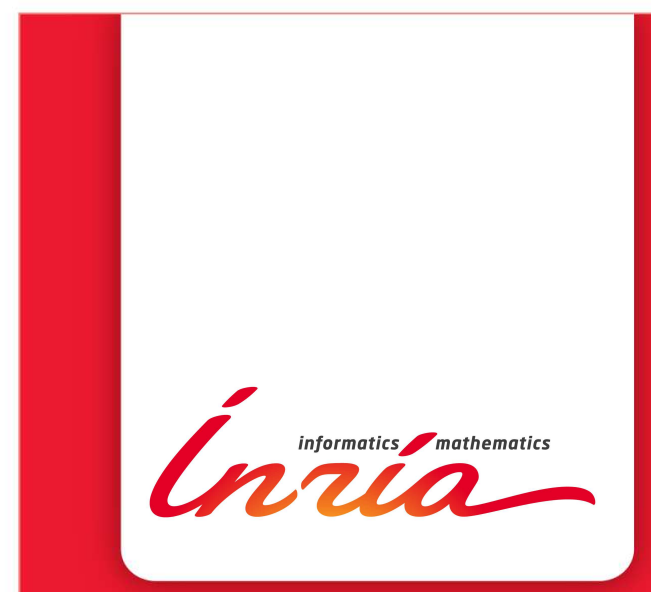

\section{Sensitivity analysis for} the outages of nuclear power plants

Kengy Barty, J. Frédéric Bonnans, Laurent Pfeiffer

RESEARCH 



\title{
inzián
}

\section{Sensitivity analysis for the outages of nuclear power plants}

\author{
Kengy Barty ${ }^{*}$, J. Frédéric Bonnans ${ }^{\dagger}$, Laurent Pfeiffer ${ }^{\dagger}$ \\ Project-Team Commands \\ Research Report $\mathrm{n}^{\circ} 7884$ - February 2012 - 29 pages
}

\begin{abstract}
Nuclear power plants must be regularly shut down in order to perform refueling and maintenance operations. The scheduling of the outages is the first problem to be solved in electricity production management. It is a hard combinatorial problem for which an exact solving is impossible.

Our approach consists in modelling the problem by a two-level problem. First, we fix a feasible schedule of the dates of the outages. Then, we solve a low-level problem of optimization of elecricity production, by respecting the initial planning. In our model, the low-level problem is a deterministic convex optimal control problem.

Given the set of solutions and Lagrange multipliers of the low-level problem, we can perform a sensitivity analysis with respect to dates of the outages. The approximation of the value function which is obtained could be used for the optimization of the schedule with a local search algorithm.
\end{abstract}

Key-words: Sensitivity analysis, nuclear power plants, optimal control, Pontryagin's principle.

* EDF R\&D, 92141 Clamart, France (kengy.barty@edf.fr)

$\dagger$ INRIA-Saclay and CMAP, École Polytechnique, 91128 Palaiseau, France (frederic.bonnans@inria.fr)

¥ INRIA-Saclay and CMAP, École Polytechnique, 91128 Palaiseau, France (laurent.pfeiffer@polytechnique.edu)

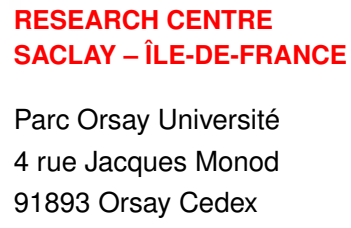




\section{Analyse de sensibilité pour les arrêts des centrales nucléaires}

Résumé : Les centrales nucléaires doivent être régulièrement arrêtées afin de réaliser des opérations de maintenance et de rechargement en combustible nucléaire. La planification de ces arrêts constitue le premier problème à résoudre en gestion de la production d'électricité. C'est un problème combinatoire difficile qui ne peut être résolu exactement.

Notre approche consiste à modéliser ce problème par un problème à deux niveaux. Tout d'abord, nous fixons un calendrier admissible des dates des arrêts des centrales. Puis, nous résolvons un sous-problème de production d'électricité, en respectant le calendrier initial. Dans notre modèle, ce sous-problème est un problème de contrôle optimal déterministe et convexe.

Etant donnés les solutions et multiplicateurs de Lagrange du sous-problème, nous pouvons réaliser une analyse de sensibilité par rapport aux dates des arrêts. L'approximation de la fonction valeur que nous obtenons devrait permettre de mettre en place un algorithme de recherche locale pour l'optimisation de ces dates d'arrêts.

Mots-clés : Analyse de sensibilité, centrales nucléaires, contrôle optimal, principe de Pontryagine. 


\section{Introduction}

Energy generation in France is a competitive market, whereas transportation and distribution are monopolies. Electric utilities generate electricity from hydro reservoirs, fossil energy (coal, gas), atom (nuclear fission process) and to a small extent from wind farms, solar energy or run of river plant without pondage. This energy mix provides enough power and flexibility to match energy demand in any circumstances. Hydro power stations are managed in order to remove peaks on the load curve during peak-hours, whereas thermal power stations supply base load energy. Due to their capacity generation and their production cost as well, the base load part is mainly supported by nuclear power stations.

Nuclear facilities are subject to various constraints, which induces a variation of the availability of nuclear energy. Some events may occur randomly during the operating period and cause forced outages. This is why outages must be planned by the producer in order to perform maintenance and refuelling operations of the fleet of nuclear power stations and in order to avoid a dramatical decrease of the nuclear availability. Thermal power stations, using expensive resources such as coal or gas, enable to compensate a lack of nuclear energy. These supplementary costs, due to the nuclear unavailability must be minimized when a schedule of the outages is planned.

Each power station has its scheduling variables, which are submitted to local and coupling constraints as well. There are different constraints in the scheduling of outages of power plants: on the minimum spacing, on the maximum overlapping between outages, and on the number of outages in parallel. For operating purposes, the decision to stop a power station for maintenance has to be forecast far ahead. Hence scheduling decisions are modelled as "open-loop" decisions, which means that they do not depend on the consumption scenario.

Given the planning of outages, the low-level problem of electricity production can be described by a discrete time dynamic and stochastic optimization problem. The overall optimization problem is a large scale, mixed integer stochastic problem. We refer to [2, 5, 6, 7] for precise descriptions of this problem. At Electricité de France, the numerical resolution of this problem uses local search algorithms in order to improve the current planned program. Numerous slight modifications are performed around the current program and the most profitable determines the next program. The computational burden to solve this problem is heavy, reducing it is a challenging task.

In this paper, we perform a sensitivity analysis of the electricity production problem when the integer parameters defining the scheduling of the outages are set. We provide a first-order expansion of the value of this low-problem, with respect to the dates of the outages. For the sake of simplicity, the low-level problem is a convex deterministic optimal control problem with continuous time. We do not consider the combinatorial side of the problem.

In the first section, we discuss the structure of solutions to the low-level problem, which are not unique in general. In the second section, we realize the sensitivity analysis by using a well suited time reparameterization. We obtain a formula for the directional derivatives of the value function using the opposite of the jumps of the true Hamiltonian at the times of beginning or end of the outages. It is based on the set of Lagrange multipliers, which we describe precisely. The result is an application of a theorem of [1]. The technical aspects related to the theorem such as the proof of qualification or the proof of convergence of the solutions to the perturbed problems are postponed in the third section. 


\section{Study of the reference problem}

In this first part, we study the low-level problem of production management and therefore consider that the dates of the outages are fixed. In our model, we only consider one outage for each plant. Applying Pontryagin's principle, we study the particular structure of the optimal controls, which are not unique in general.

\subsection{Notations, model and mathematical hypotheses}

The main notations for the problem are the following:

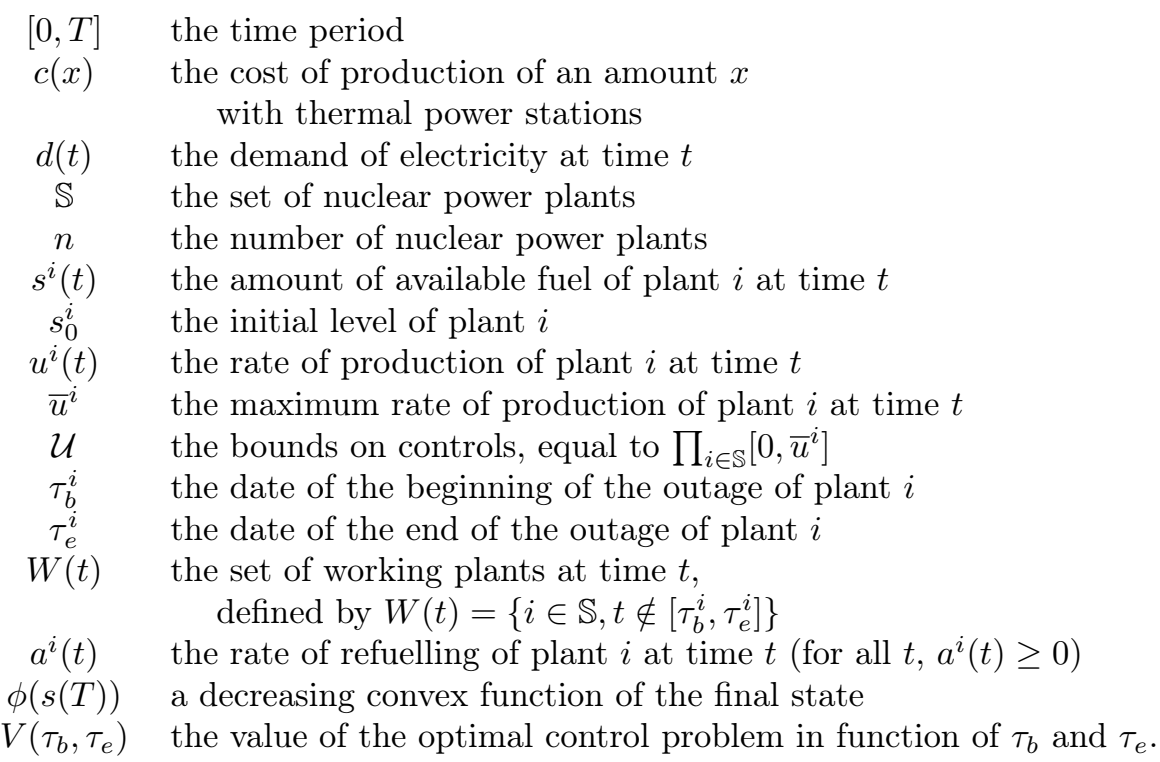

The optimal control problem $\left[\mathcal{P}\left(\tau_{b}, \tau_{e}\right)\right]$ is

$$
\begin{aligned}
& V\left(\tau_{b}, \tau_{e}\right)=\min _{u, s} \int_{0}^{T} c\left(d(t)-\sum_{i \in W(t)} u^{i}(t)\right) \mathrm{d} t+\phi(s(T)), \\
& \text { s.t. } \forall i \in \mathbb{S}, \quad \dot{s}^{i}(t)=-u^{i}(t)+a^{i}(t) \mathbf{1}_{\left[\tau_{b}^{i}, \tau_{e}^{i}\right]}(t), \quad \text { for a. a. } t \text {, } \\
& 0 \leq u^{i}(t) \leq \bar{u}^{i} \\
& \text { for a. a. } t \text {, } \\
& s^{i}(0)=s_{0}^{i} \text {, } \\
& s^{i}\left(\tau_{b}^{i}\right)=0 \text {, } \\
& s^{i}(T) \geq 0 \text {, }
\end{aligned}
$$

where $u \in L^{\infty}\left(0, T ; \mathbb{R}^{n}\right)$ and $s \in W^{1, \infty}\left(0, T ; \mathbb{R}^{n}\right)$.

The dynamic of the stocks of fuel is clear from the differential equation: the stock $s^{i}$ decreases at rate $u^{i}(t)$ during the time period and increases at rate $a^{i}(t)$ during the time of outage. The argument of the cost function $c$ is the amount of energy which is not produced with nuclear power plants in order to satisfy the demand. This energy is produced with other types of power stations, which are more expensive. In our model, we also allow the total production to be greater than the demand.

Note that for optimal solutions, we will obtain that for all $i$ in $\mathbb{S}$, for almost all $t$ in $\left[\tau_{b}^{i}, \tau_{e}^{i}\right]$, the control $u^{i}(t)$ is equal to 0 , see (4). 
Mathematical hypotheses For our study, we suppose that the following hypotheses are satisfied:

- the cost functions $c(x)$ and $\phi(s)$, the demand $d(t)$ and the rate of refuelling $a(t)$ are continuously differentiable functions,

- the cost function $c(x)$ is strongly convex with parameter $\alpha$ on $\mathbb{R}$,

- the final cost function $\phi(s)$ is strictly convex on $\mathbb{R}_{+}^{n}$ and for all $s$ in $\mathbb{R}_{+}^{n}$, for all $i$ in $\mathbb{S}$,

$$
D_{s^{i}} \phi(s)<0 .
$$

Feasibility of the problem The problem has a feasible control with a feasible trajectory associated if and only if, for all $i$ in $\mathbb{S}$,

$$
\tau_{b}^{i} \cdot \bar{u}^{i} \geq s_{0}^{i} .
$$

Moreover, we can prove the existence of an optimal solution in this case. It follows from the boundedness of the controls and the convexity of the cost functions, see lemma 17. In the sequel, we will assume that the following qualification condition is satisfied: for all $i$ in $\mathbb{S}$,

$$
\tau_{b}^{i}>0, \quad \bar{u}^{i}>0, \quad \tau_{b}^{i} \cdot \bar{u}^{i}>s_{0}^{i}, \quad \text { and } \quad \int_{\tau_{b}^{i}}^{\tau_{e}^{i}} a^{i}(t) \mathrm{d} t>0 .
$$

Note that this last integral is equal to $s^{i}\left(\tau_{e}^{i}\right)$. This hypothesis will enable us to prove an abstract qualification condition, needed to apply Pontryagin's principle and to realize the sensitivity analysis (see lemma 18 .

\subsection{Study of the optimal controls}

This subsection is dedicated to the study of an optimal control $u(t)$, which minimizes the Hamiltonian for almost all $t$. For our problem, the Hamiltonian has the particularity to be independent on the state $s$.

Let us denote by $p$ the costate associated with $s$. Given a subset $W$ of $\mathbb{S}$, we define the Hamiltonian of the system by

$$
H_{W}(t, u, p)=c\left(d(t)-\sum_{i \in W} u^{i}\right)+\sum_{i \in \mathbb{S}} p^{i}\left(-u^{i}+a^{i}(t) \mathbf{1}_{i \notin W}\right)
$$

for $t$ in $[0, T], u$ in $\mathcal{U}$ and $p$ in $\mathbb{R}^{n}$. The subscript $W$ refers to the set of working plants. Notice that it does not depend on the state $s$.

Proposition 1 (Pontryagin's principle). If hypothesis QC holds, then for all optimal solution $(u, s)$, there exists a costate $t \mapsto p(t) \in \mathbb{R}^{n}$ such that for all $i$ in $\mathbb{S}$,

- $p^{i}(t)$ is a step function, taking two values $p^{i}(0)$ and $p^{i}(T)$ on the intervals $\left[0, \tau_{b}^{i}\right)$ and $\left(\tau_{b}^{i}, T\right]$ respectively

- $p^{i}(T) \leq D_{s^{i}} \phi(s(T))$ and $p^{i}(T)=D_{s^{i}} \phi(s(T))$ if $s^{i}(T)>0$

and such that for almost all $t$ in $[0, T]$, the control minimizes the Hamiltonian:

$$
H_{W(t)}(t, u(t), p(t))=\min _{v \in \mathcal{U}} H_{W(t)}(t, v, p(t)) .
$$


Proof. In lemma 18, we prove that hypothesis (QC) implies Robinson's qualification condition (RQC). This condition enables us to apply Pontryagin's principle for systems with a final-state constraint, see [4, section 2.4.1, theorem 1] for a proof. For our problem, each state variable $s^{i}$ can be decomposed into two state variables, one describing the dynamic of the stock before its outage, one describing its dynamic after. This is why we can view the constraint $s\left(\tau_{b}^{i}\right)=0$ as a final-state constraint. The costate $p$ is a step function because nor the dynamic, neither the cost function depend on the state. The discontinuity of the coordinate $p^{i}$ at time $\tau_{b}^{i}$ is due to the state constraint $s\left(\tau_{b}^{i}\right)=0$.

In the sequel, we will consider that a costate is an element of $\mathbb{R}^{2 n}$ which is characterized by its values $p(0)$ and $p(T)$ at times 0 and $T$. For all $p=\left(p_{0}, p_{T}\right)$ in $\mathbb{R}^{2 n}$, we associate the costate function defined by

$$
p^{i}(t)=\left\{\begin{array}{ll}
p^{i}(0) & \text { if } t \in\left[0, \tau_{b}^{i}\right), \\
p^{i}(T) & \text { if } t \in\left(\tau_{e}^{i}, T\right],
\end{array} \quad \forall i \in \mathbb{S} .\right.
$$

We assign no value to $p^{i}$ at time $\tau_{b}^{i}$. However, we will use the following notations in the sequel: if plant $i$ is the only plant to start an outage at time $t=\tau_{b}^{i}, p\left(t^{-}\right)$and $p\left(t^{+}\right)$are such that for all $j \neq i$,

$$
p^{j}\left(t^{-}\right)=p^{j}\left(t^{+}\right)=p^{j}(t)
$$

and such that

$$
p^{i}\left(t^{-}\right)=p^{i}(0), \quad \text { and } \quad p^{i}\left(t^{+}\right)=p^{i}(T) .
$$

Given $p$ in $\mathbb{R}^{n}$ and $t$ in $[0, T]$, the optimization problem associated with the minimization of the Hamiltonian at time $t$ is the following:

$$
\min _{v \in \mathcal{U}} c\left(d(t)-\sum_{i \in W(t)} v^{i}\right)+\sum_{i \in \mathbb{S}} p^{i}(t)\left(-v^{i}+a^{i}(t) \mathbf{1}_{\left[\tau_{b}^{i}, \tau_{e}^{i}\right]}(t)\right) .
$$

As we can see, the term $\sum_{i \in \mathbb{S}} p^{i}(t) a^{i}(t) \mathbf{1}_{\left[\tau_{b}^{i}, \tau_{e}^{i}\right]}(t)$ does not play any role here. Moreover, we can decompose the problem by introducing an additional variable $\mu$ for the total production, the sum $\sum_{i \in W(t)} v^{i}$. For a subset $W$ of $\mathbb{S}$, let us set

$$
u^{W}=\sum_{i \in W} \bar{u}^{i}
$$

and let us define, for $\mu$ in $\left[0, u^{W}\right]$,

$$
\xi_{W}(\mu, p)=\min _{\substack{v \in \mathcal{U}, \sum_{i \in W} v^{i}=\mu}} \sum_{i \in W}-p^{i} v^{i} .
$$

Now, we can focus on the following one-dimensional problem:

$$
\min _{0 \leq \mu \leq u^{W(t)}} c(d(t)-\mu)+\xi_{W(t)}(\mu, p) .
$$

Let $u$ be an optimal solution to the problem and let $p$ be an associated costate. If at time $t$, plant $i$ does not belong to $W(t)$, then it means that $t>\tau_{b}^{i}$ and thus

$$
p^{i}(t)=p^{i}(T) \leq D_{s^{i}} \phi(s(T))<0 .
$$

As a consequence, it is clear that if $v$ is a solution to problem $P_{t, p}$ with $p=p(t)$, then $v^{i}=0$ for all $i \notin W(t)$ and $\sum_{i \in W(t)} v^{i}$ is a solution to $P_{t, p}^{\prime}$. Conversely, if $\mu$ is a solution to $P_{t, p}^{\prime}$ then there 
exists a solution $v$ to $P_{t, p}$ such that $\sum_{i \in W(t)} v^{i}=\mu$. Since for all $i$ in $\mathbb{S}$, for all $t \geq \tau_{b}^{i}, p^{i}(t)<0$, we obtain that

$$
u^{i}(t)=0, \quad \text { for a. a. } t \in\left[\tau_{b}^{i}, \tau_{e}^{i}\right] .
$$

Problem $P_{t, p}^{\prime}$ has an economic interpretation. Producing at time $t$ at a rate $u$ has a consequence on the dynamic of the state after time $t$. This is represented by the function $\xi_{W}(t)(\mu, p)$. In some sense, the real numbers $-p^{1}, \ldots,-p^{n}$ are the marginal prices associated with the production at time $t$. Problem $P_{t, p}^{\prime}$ takes into account both the cost function $c(d(t)-\mu)$ and the cost of production $\xi_{W(t)}(\mu, p)$.

Notations In the next three lemmas, we focus on problem $P_{t, p}^{\prime}$. We fix $t$ in $[0, T]$ and $p$ in $\mathbb{R}^{n}$. Let us denote by $K$ the cardinal of $\left\{p^{i}, i \in W(t)\right\}$. In the sequel, keep in mind that it may be possible that $p^{i}=p^{j}$ for some $i$ and $j$ in $W(t)$. In this case, the corresponding value $p^{i}=p^{j}$ is counted only once and then $K<|W(t)|$. We consider the mapping $\sigma$ from $\{1, \ldots, K\}$ to $\mathcal{P}(W(t))$ (the power set of $W(t))$ uniquely defined by:

(i) $\forall k \in\{1, \ldots, K\}, \forall i \in \sigma(k), \forall j \in W(t), p^{j}=p^{i} \Rightarrow j \in \sigma(k)$.

This common value will be denoted by $p_{k}$.

(ii) $\forall k, l \in\{1, \ldots, K\}, k<l \Rightarrow p_{k}>p_{l}$.

This mapping is nothing but a decreasing ordering of the coordinates of $p$ involved in the definition of $\xi_{W}$. We also set, for $k$ in $\{1, \ldots, K\}$

$$
U_{k}=\sum_{l=1}^{k} \sum_{i \in \sigma(l)} \bar{u}^{i}
$$

and $U_{0}=0$. In the sequel, indexes $i$ and $j$ will be elements of $\mathbb{S}$ and will appear at the top, whereas indexes $k$ and $l$ will be elements of $\{1, \ldots, K\}$ and will appear at the bottom.

Let $W$ be a subset of $\mathbb{S}$, the function $\mu \mapsto \xi_{W}(\mu, p)$ is piecewise affine and convex. We make its value explicit on $\left[0, U_{K}\right]$ :

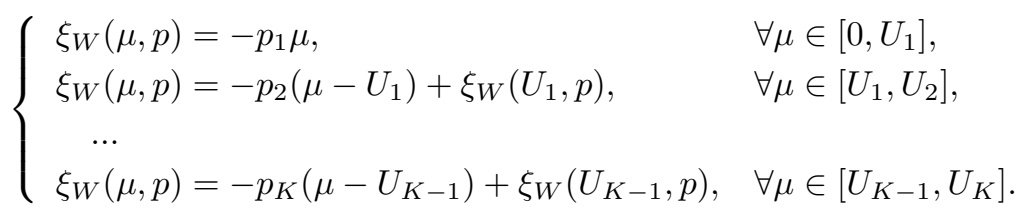

Lemma 2. On an interval of time $[a, b]$ where $W(t)$ is constant, there is an ordering in the use of the fuel: we begin by using the power of the plants of greatest costate. More precisely, if $i$ and $j$ are in $W(t)$ and are such that $-p^{i}(t)<-p^{j}(t)$, if $u(t)$ is an optimal control, then for almost all $t$ in $[a, b]$,

$$
\left(u^{j}(t)>0\right) \Rightarrow\left(u^{i}(t)=\bar{u}^{i}\right)
$$

or, equivalently,

$$
\left(u^{i}(t)<\bar{u}^{i}\right) \Rightarrow\left(u^{j}(t)=0\right) .
$$

Proof. This follows from the expression of $\xi_{W}(\mu, p)$ given by (7). Recall that when $W(t)$ is constant on an interval of time, there is no beginning or ending of outage and $p(t)$ is also constant on this interval. 
The interpretation of the lemma is the following: if $-p^{i}<-p^{j}$, then plant $i$ is cheaper than plant $j$ at time $t$ and should be used first. The next lemma gives the necessary optimality conditions of problem $P_{t, p}^{\prime}$

Lemma 3. Problem $P_{t, p}^{\prime}$ has a unique solution on $\left[0, U_{K}\right]$. Let $\mu$ be this solution and let us distinguish four cases.

1. There exists $k$ in $\{1, \ldots, K\}$ such that $\mu \in] U_{k-1}, U_{k}[$. Then,

$$
c^{\prime}(d(t)-\mu)=-p_{k} .
$$

2. There exists $k$ in $\{0, \ldots, K\}$ such that $\mu=U_{k}$.

(a) If $k \in\{1, \ldots, K-1\}$,

$$
-p_{k} \leq c^{\prime}\left(d(t)-U_{k}\right) \leq-p_{k+1}
$$

(b) If $k=0$,

$$
c^{\prime}(d(t)-0) \leq-p_{1}
$$

(c) If $k=K$,

$$
-p_{K} \leq c^{\prime}\left(d(t)-U_{K}\right)
$$

Proof. The function $\mu \mapsto c(d(t)-\mu)+\xi_{W(t)}(\mu, p(t))$ is continuous and defined on a bounded interval, whence the existence of the solution. Furthermore, this function is strictly convex, since $c$ is so. The uniqueness of the solution follows. For the optimality conditions, we use the assumption of differentiability of $c$ and the explicit formula of $\xi_{W(t)}(\mu, p)$ given by (7).

The goal of the next lemma is to give a characterization of the solutions to problem $P_{t, p}^{\prime}$ in function of $d(t)$. We denote by $k^{b}$ and $k^{\sharp}$ the smallest indexes such that

$$
\lim _{x \rightarrow-\infty} c^{\prime}(x)<-p_{k^{b}} \quad \text { and } \quad-p_{k^{\sharp}}<\lim _{x \rightarrow+\infty} c^{\prime}(x) \text { respectively. }
$$

For all $k$ in $\left\{k^{b}, \ldots, k^{\sharp}\right\}$, we set

$$
\left\{\begin{array}{l}
d_{k}^{b}=c^{\prime-1}\left(-p_{k}\right)+U_{k-1}, \\
d_{k}^{\sharp}=c^{\prime-1}\left(-p_{k}\right)+U_{k} .
\end{array}\right.
$$

We also set

$$
d_{k^{b}-1}^{\sharp}=-\infty \quad \text { and } \quad d_{k^{\sharp}+1}^{b}=+\infty .
$$

We have

$$
d_{k^{b}-1}^{\sharp}<d_{k^{b}}^{b}<d_{k^{b}}^{\sharp}<d_{k^{b}+1}^{b}<\cdots<d_{k^{\sharp}}^{b}<d_{k^{\sharp}}^{\sharp}<d_{k^{\sharp}+1}^{b} .
$$

Now, we can express the optimal solution $\mu$ in function of $d(t)$.

Lemma 4. Let us consider two cases.

1. If for some $k$ in $\left\{k^{b}, \ldots, k^{\sharp}\right\}, d_{k}^{b} \leq d(t) \leq d_{k}^{\sharp}$, then

$$
\begin{aligned}
& \mu=d(t)-c^{\prime-1}\left(-p_{k}\right), \\
& u^{i}=\bar{u}^{i}, \forall i \in \sigma(l), \quad l<k, \\
& \sum_{i \in \sigma(k)} u^{i}=d(t)-c^{\prime-1}\left(-p_{k}\right)-U_{k-1}, \\
& u^{i}=0, \forall i \in \sigma(l), \quad l>k .
\end{aligned}
$$


2. If for some $k$ in $\left\{k^{b}-1, \ldots, k^{\sharp}\right\}, d_{k}^{\sharp} \leq d(t) \leq d_{k+1}^{b}$, then

$$
\begin{aligned}
& \mu=U_{k}, \\
& u^{i}=0, \forall i \in \sigma(l), l>k, \\
& u^{i}=\bar{u}^{i}, \forall i \in \sigma(l), \quad l \leq k .
\end{aligned}
$$

Proof. Since the problem is convex, it suffices to check the necessary optimality conditions detailed in lemma 3. For the first case, the condition satisfied is (8). In the second case, if $k=0$, the condition satisfied is $(10)$, if $k=K$, the condition satisfied is (11), otherwise, the condition satisfied is 9 .

Remark 5. In lemma 4, we see that the coefficients $d_{k}^{b / \sharp}$ play an important role, since they enable us to compute the optimal solutions to problem $P_{t, p}$. Keep in mind that these coefficients depend on $p(t)$. As a consequence, they have to be viewed as step functions of time.

We state now a uniqueness property of the optimal controls.

Lemma 6. Let $\left(u_{1}, s_{1}\right)$ and $\left(u_{2}, s_{2}\right)$ be two optimal solutions. Then, for almost all $t$ in $[0, T]$, $\sum_{i \in \mathbb{S}} u_{1}^{i}(t)=\sum_{i \in \mathbb{S}} u_{2}^{i}(t)$ and $s_{1}(T)=s_{2}(T)$.

Proof. It is well-known that for a convex optimization problem, if the cost function is strictly convex with respect to one of the optimization variables, then the value of this variable is unique at the optimum. For our problem, since $c$ and $\phi$ are strictly convex, we have that $\sum_{i \in W(t)} u_{1}^{i}(t)=$ $\sum_{i \in W(t)} u_{2}^{i}(t)$ for almost all $t$ in $[0, T]$ and $s_{1}(T)=s_{2}(T)$. Since $u_{1}(t)=u_{2}(t)=0$ for almost all $t$ in $\left[\tau_{b}^{i}, \tau_{e}^{i}\right]$, we finally obtain that $\sum_{i \in \mathbb{S}} u_{1}^{i}(t)=\sum_{i \in \mathbb{S}} u_{2}^{i}(t)$ for almost all $t$.

Remark 7. While the sum of the controls is unique, there may be several differents optimal controls. This happens when there are at least two plants $i$ and $j$ for which $p^{i}(t)=p^{j}(t)$ on a subinterval of $[0, T]$. If the demand satisfies strictly the inequalities of the first case of lemma 4 . then the problem of minimization of the Hamiltonian, $P_{t, p}$, has several optimal solutions and the general problem has equally, in general, several solutions. 


\section{Sensitivity analysis}

\subsection{Theoretical material}

In this subsection, we state an abstract general theorem for the sensitivity analysis of a convex problem. Consider the general parameterized problem

$$
V(y)=\min _{x \in X} f(x, y), \text { subject to } G(x, y) \in K,
$$

in which $y$ stands for the perturbation parameter and belongs to a space $Y$. The functions $f$ and $G$ are supposed to be continuously differentiable with respect to $x$ and $y . K$ is a closed convex subset of a space $Z$. The spaces $X, Y$ and $Z$ are Banach spaces. We fix a reference value $y_{0}$ for $y$. Let $x$ be a feasible point of the reference problem with $y=y_{0}$. We say that Robinson's qualification condition holds at $x$ if there exists $\varepsilon>0$ such that

$$
\varepsilon B_{Z} \subset G\left(x, y_{0}\right)+D_{x} G\left(x, y_{0}\right) X-K,
$$

where $B_{Z}$ is the unit ball of $Z$. For $\lambda$ in $Z^{*}$, we define the Lagrangian by

$$
L(x, \lambda, y)=f(x, y)+\langle\lambda, G(x, y)\rangle .
$$

In a general framework, for a solution $x_{0}$ to the optimization problem with $y=y_{0}$, the set of Lagrange multipliers $\Lambda\left(x_{0}, y_{0}\right)$ is defined by

$$
\Lambda\left(x_{0}, y_{0}\right)=\left\{\lambda \in Z^{*}, D_{x} L\left(x_{0}, \lambda, y_{0}\right)=0, \lambda \in N_{K}\left(G\left(x_{0}, u_{0}\right)\right\} .\right.
$$

We suppose now that the reference problem $P_{y_{0}}$ is convex, following definition 2.163 of [1]. Problems, like our application problem, with a convex cost function, linear equality constraints, and finite convex inequality constraints are convex. For a convex problem, the set of Lagrange multipliers is the set of solutions of a dual problem which does not depend on the choice of the (primal) solution $x_{0}$. Therefore, $\Lambda\left(x_{0}, y_{0}\right)$ does not depend on $x_{0}$ and we simply write $\Lambda\left(y_{0}\right)$.

The following theorem establishes a differentiability property of the value function of the problem $V(y)$. See [1, definition 2.45] for a definition of the Hadamard differentiability.

Theorem 8. Consider a reference value $y_{0}$. Suppose that:

1. Problem $P_{y_{0}}$ is convex and has a non-empty set of optimal solutions $S\left(y_{0}\right)$.

2. Robinson's qualification condition holds at all $x_{0}$ in $S\left(y_{0}\right)$.

3. For all sequence $\left(y_{k}\right)_{k}$ converging to $y_{0}$, problem $P_{y_{k}}$ possesses an optimal solution $x_{k}$ such that, for all $\lambda$ in $\Lambda\left(y_{0}\right)$, for all sequence $\left(y_{k}^{\prime}\right)_{k}$ satisfying $y_{k}^{\prime} \in\left[y_{0}, y_{k}\right]$, one has:

$$
D_{y} L\left(x_{0}, \lambda, y_{0}\right) \text { is a limit point of } D_{y} L\left(x_{k}, \lambda, y_{k}^{\prime}\right) \text {. }
$$

Then the optimal value function $V$ is Hadamard directionally differentiable at $y_{0}$ in any direction $w$ and

$$
V^{\prime}\left(y_{0}, w\right)=\inf _{x \in S\left(y_{0}\right)} \sup _{\lambda \in \Lambda\left(y_{0}\right)} D_{y} L\left(x, \lambda, y_{0}\right) w .
$$

Proof. This theorem is a direct extension of [1, theorem 4.24], which was originally proved in 3]. Note first that since Robinson's qualification condition holds and since there exist optimal solutions, the set $\Lambda\left(y_{0}\right)$ of Lagrange multipliers is nonempty and thus, the expression of the 
directional derivative (14) is finite. It is proved in [1, proposition 4.22] that under the directional regularity condition, for any $y(\alpha)=y_{0}+\alpha w+o(\alpha)$, the following holds:

$$
\limsup _{\alpha \downarrow 0} \frac{V(y(\alpha))-V\left(y_{0}\right)}{\alpha} \leq \inf _{x \in S\left(y_{0}\right)} \sup _{\lambda \in \Lambda\left(y_{0}\right)} D_{y} L\left(x, \lambda, y_{0}\right) w
$$

The directional regularity condition is implied by Robinson's qualification condition, see 1 , theorem 4.9].

Let $\alpha_{n} \downarrow 0$, let $y_{n}=y_{0}+\alpha_{n} w+o\left(\alpha_{n}\right)$ and let $\left(x_{n}\right)_{n}$ be the sequence of solutions such that hypothesis (3) of the theorem is satisfied. Extracting a subsequence if necessary, we can suppose that $\left(x_{n}\right)_{n}$ converges to $x_{0}$ in $S\left(y_{0}\right)$. Let $\lambda$ be in $\Lambda\left(y_{0}\right)$. Since $\Lambda\left(y_{0}\right) \subset N_{K}\left(G\left(x_{0}, y_{0}\right)\right)$, and hence

$$
\left\langle\lambda, G\left(x_{n}, y_{n}\right)-G\left(x_{0}, y_{0}\right)\right\rangle \leq 0,
$$

we have

$$
f\left(x_{n}, y_{n}\right)-f\left(x_{0}, y_{0}\right) \geq L\left(x_{n}, \lambda, y_{n}\right)-L\left(x_{0}, \lambda, y_{0}\right) .
$$

By convexity of $P_{y_{0}}$, the first order optimality conditions imply that $x_{0}$ belongs to

$$
\arg \min _{x \in X} L\left(x, \lambda, y_{0}\right)
$$

and hence

$$
L\left(x_{n}, \lambda, y_{0}\right) \geq L\left(x_{0}, \lambda, y_{0}\right) .
$$

Since $V\left(y_{n}\right)=f\left(x_{n}, y_{n}\right)$ and by the mean value theorem and continuity of $L(x, \lambda, y)$, we obtain from 16 that, for some $y_{n}^{\prime}$ in $\left[y_{0}, y_{n}\right]$,

$$
\begin{aligned}
V\left(y_{n}\right)-V\left(y_{0}\right) & \geq L\left(x_{n}, \lambda, y_{n}\right)-L\left(x_{n}, \lambda, y_{0}\right) \\
& =\alpha_{n} D_{y} L\left(x_{n}, \lambda, y_{n}^{\prime}\right) w \\
& =\alpha_{n} D_{y} L\left(x_{0}, \lambda, y_{0}\right) w+o(\alpha),
\end{aligned}
$$

since by assumption,

$$
D_{y} L\left(x_{n}, \lambda, y_{n}^{\prime}\right) \rightarrow D_{y} L\left(x_{0}, \lambda, y_{0}\right)
$$

As a consequence, since $\lambda$ was arbitrary,

$$
\liminf _{n \rightarrow \infty} \frac{V\left(y_{n}\right)-V\left(y_{0}\right)}{\alpha_{n}} \geq \sup _{\lambda \in \Lambda\left(y_{0}\right)} D_{y} L\left(x_{0}, \lambda, y_{0}\right) \geq \inf _{x \in S\left(y_{0}\right)} \sup _{\lambda \in \Lambda\left(y_{0}\right)} D_{y} L\left(x, \lambda, y_{0}\right) w
$$

Combining (15) and (17), we obtain that for any $y(\alpha)=y_{0}+\alpha w+o(\alpha)$,

$$
\lim _{\alpha \downarrow 0} \frac{V(y(\alpha))-V\left(y_{0}\right)}{\alpha}=\inf _{x \in S\left(y_{0}\right)} \sup _{\lambda \in \Lambda\left(y_{0}\right)} D_{y} L\left(x, \lambda, y_{0}\right) w,
$$

as was to be proved.

\subsection{Expression of the directional derivatives}

In this subsection, we give a sensitivity formula for problem $\sqrt{\mathcal{P}\left(\tau_{b}, \tau_{e}\right)}$. We show that the value function is Hadamard directionally differentiable if all the dates of the outages are different. When differentiating the value function with respect to one variable, the result obtained is the jump of the reduced Hamiltonian at the reference time of the variable. 
Time reparameterization Theorem 8 cannot be applied directly to our application problem. Indeed, in its formulation, the cost function and the dynamic are not continuously differentiable with respect to $\tau_{b}$ and $\tau_{e}$. For example, if we try to differentiate the cost function with respect to the variable $\tau_{e}^{j}$, we obtain the following derivative,

$$
c\left(\tau_{e}^{j}, d\left(\tau_{e}^{j}\right)-\sum_{i \in W} u^{i}\left(\tau_{e}^{j}\right)\right)-c\left(\tau_{e}^{j}, d\left(\tau_{e}^{j}\right)-\sum_{i \in W \cup\{j\}} u^{i}\left(\tau_{e}^{j}\right)\right),
$$

where $W$ is the set of working plants at time $\tau_{e}^{j}$ ( $j$ being excluded of $W$ ). This expression does not make sense, since the control is only in $L^{\infty}\left(0, T ; \mathbb{R}^{n}\right)$, thus, we cannot define its value at time $\tau_{e}^{j}$.

However, if we perform a well suited change of variable in time, we can apply the abstract result. The change of variable that we use can be realized if and only if the following hypothesis holds:

$$
\text { For all } i \text { and } j \text { in } \mathbb{S} \text { such that } i \neq j, \tau_{b}^{i} \neq \tau_{b}^{j}, \tau_{e}^{i} \neq \tau_{e}^{j} \text { and } \tau_{b}^{i} \neq \tau_{e}^{j} \text {. }
$$

We begin by computing $D_{\tau_{e}^{j}} V\left(\tau_{b}, \tau_{e}\right)$. We consider a nuclear power plant $j$ and we denote by $\tau_{0}$ the reference value of $\tau_{e}^{j}$ and by $(u, s)$ a solution with a costate $p$, for the reference problem with $\tau_{e}^{j}=\tau_{0}$. As a consequence of $(\mathrm{H})$, we get :

None of the plants, except $j$, begins or ends its outage at time $\tau_{0}$.

Let us consider two times $t_{1}$ and $t_{2}$ such that $t_{1}<\tau_{0}<t_{2}$ and such that they are sufficiently close to $\tau_{0}$ so that none of the plants (except $j$ ) begins or ends an outage during $\left[t_{1}, t_{2}\right]$. The idea of the change of variable is to fix the time of the discontinuity due to the end of the outage. We set, for all $t^{\prime}$ in $[0, T]$,

$$
\theta_{\tau_{e}^{j}}\left(t^{\prime}\right)= \begin{cases}t_{1}+\frac{\tau_{e}^{j}-t_{1}}{\tau_{0}-t_{1}}\left(t^{\prime}-t_{1}\right), & \text { if } t^{\prime} \in\left[t_{1}, \tau_{0}\right] \\ t_{2}-\frac{t_{2}-\tau_{e}^{j}}{t_{2}-\tau_{0}}\left(t_{2}-t^{\prime}\right), & \text { if } t^{\prime} \in\left[\tau_{0}, t_{2}\right] \\ t^{\prime}, & \text { otherwise. }\end{cases}
$$

We perform the change of variable $t=\theta_{\tau_{e}^{j}}\left(t^{\prime}\right)$. See figure 1 for an illustration of the change of variable. It is well defined for $\tau_{e}^{j}$ in $\left(t_{1}, t_{2}\right)$. We denote by $W$ the set of working plants on the interval $\left[t_{1}, t_{2}\right]$. By convention, $j$ does not belong to $W$. The new optimal control problem 


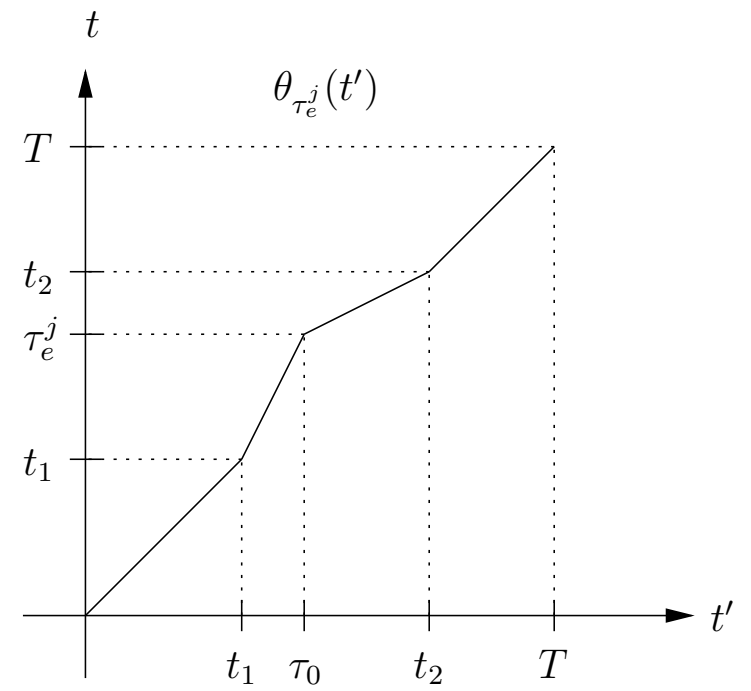

Figure 1: Change of variable associated with the perturbation of $\tau_{e}^{j}$.

$\mathcal{P}^{\prime}\left(\tau_{e}^{j}\right)$ to be solved is

$$
\begin{aligned}
& V\left(\tau_{e}^{j}\right)=\min _{u, s}\left[\int_{0}^{t_{1}} c\left(d\left(t^{\prime}\right)-\sum_{i \in W\left(t^{\prime}\right)} u^{i}\left(t^{\prime}\right)\right) \mathrm{d} t^{\prime}\right. \\
& +\frac{\tau_{e}^{j}-t_{1}}{\tau_{0}-t_{1}} \int_{t_{1}}^{\tau_{0}} c\left(d \circ \theta_{\tau_{e}^{j}}\left(t^{\prime}\right)-\sum_{i \in W} u^{i}\left(t^{\prime}\right)\right) \mathrm{d} t^{\prime} \\
& +\frac{t_{2}-\tau_{e}^{j}}{t_{2}-\tau_{0}} \int_{\tau_{0}}^{t_{2}} c\left(d \circ \theta_{\tau_{e}^{j}}\left(t^{\prime}\right)-\sum_{i \in W \cup\{j\}} u^{i}\left(t^{\prime}\right)\right) \mathrm{d} t^{\prime} \\
& \left.+\int_{t_{2}}^{T} c\left(d\left(t^{\prime}\right)-\sum_{i \in W\left(t^{\prime}\right)} u^{i}\left(t^{\prime}\right)\right) \mathrm{d} t^{\prime}+\phi(s(T))\right], \\
& \begin{cases}\frac{\tau_{e}^{j}-t_{1}}{\tau_{0}-t_{1}}\left[-u^{i}\left(t^{\prime}\right)+a^{i} \circ \theta_{\tau_{e}^{j}}\left(t^{\prime}\right) \mathbf{1}_{i \notin W}\right], & \text { if } t^{\prime} \in\left[t_{1}, \tau_{0}\right], \\
\frac{t_{2}-\tau_{e}^{j}}{t_{2}-\tau_{0}}\left[-u^{i}\left(t^{\prime}\right)+a^{i} \circ \theta_{\tau_{e}^{j}}\left(t^{\prime}\right) \mathbf{1}_{i \notin W \cup\{j\}}\right], & \text { if } t^{\prime} \in\left[\tau_{0}, t_{2}\right], \\
-u^{i}\left(t^{\prime}\right)+a^{i}\left(t^{\prime}\right) \mathbf{1}_{\left[\tau_{b}^{i}, \tau_{e}^{i}\right]}\left(t^{\prime}\right), & \text { otherwise, }\end{cases} \\
& 0 \leq u^{i}\left(t^{\prime}\right) \leq \bar{u}^{i} \\
& s^{i}(0)=s_{0}^{i} \text {, } \\
& s^{i}\left(\tau_{b}^{i}\right)=0 \text {, } \\
& s^{i}(T) \geq 0 \text {. }
\end{aligned}
$$

It is easy to check that problems $\sqrt{\mathcal{P}\left(\tau_{b}, \tau_{e}\right)}$ and $\sqrt{\mathcal{P}^{\prime}\left(\tau_{e}^{j}\right)}$ have the same value when $\tau_{e}^{j}$ belongs to $\left(t_{1}, t_{2}\right)$. Since for all $t^{\prime}$ in $[0, T], \theta_{\tau_{0}}\left(t^{\prime}\right)=t^{\prime}$, the original and the reparameterized problems are the same for $\tau_{e}^{j}=\tau_{0}$.

Remark 9. Notice that this reparameterization is not correct anymore when another plant begins or ends an outage at time $\tau_{0}$. Indeed, in this case, we cannot anymore identify a constant set of working plants ( $j$ let alone) in the neighborhood of time $\tau_{0}$. 
Remark 10. The Hamiltonian $H^{\prime}$ associated with the reparameterized problem is the following:

$$
H^{\prime}\left(t^{\prime}, u, p\right)=\dot{\theta}_{\tau_{e}^{j}}\left(t^{\prime}\right) H_{W\left(t^{\prime}\right)}\left(\theta_{\tau_{e}^{j}}\left(t^{\prime}\right), u, p\right)
$$

Note that the set of working plants $W\left(t^{\prime}\right)$ at time $t^{\prime}$ is defined with respect to the dates of the reference problem. Moreover, $(u, s)$ is an optimal solution to $\mathcal{P}\left(\tau_{b}, \tau_{e}\right)$ with associated costate $p$ if and only if $\left(u \circ \theta_{\tau_{e}^{j}}, s \circ \theta_{\tau_{e}^{j}}\right)$ is an optimal solution to $\mathcal{P}^{\prime}\left(\tau_{e}^{j}\right)$ with associated costate $p \circ \theta_{\tau_{e}^{j}}$.

Derivation of the Lagrangian Let $(u, s)$ be a solution to the reference problem with $\tau_{e}^{j}=\tau_{0}$, let $p$ be an associated costate. We consider a Lagrangian on the interval $\left[t_{1}, t_{2}\right]$, where the variable $\tau_{e}^{j}$ appears. Note that $p$ is constant on this interval. For the sake of simplicity, we write $p$ instead of $p\left(t^{\prime}\right)$ and $t$ instead of $t^{\prime}$. In the following Lagrangian, we only take into account the part of the cost function and the part of the dynamic associated with the interval $\left[t_{1}, t_{2}\right]$, where the perturbation happens.

$$
\begin{aligned}
& \mathcal{L}\left(u, s, p, \tau_{e}^{j}\right) \\
& =\frac{\tau_{e}^{j}-t_{1}}{\tau_{0}-t_{1}} \int_{t_{1}}^{\tau_{0}} c\left(d \circ \theta_{\tau_{e}^{j}}(t)-\sum_{i \in W} u^{i}(t)\right) \mathrm{d} t \\
& \quad+\frac{t_{2}-\tau_{e}^{j}}{t_{2}-\tau_{0}} \int_{\tau_{0}}^{t_{2}} c\left(d \circ \theta_{\tau_{e}^{j}}(t)-\sum_{i \in W \cup\{j\}} u^{i}(t)\right) \mathrm{d} t \\
& \quad+\sum_{i \in \mathbb{S}} p^{i} \int_{t_{1}}^{\tau_{0}}\left(-\dot{s}^{i}(t)+\frac{\tau-t_{1}}{\tau_{0}-t_{1}}\left(-u^{i}(t)+a^{i} \circ \theta_{\tau_{e}^{j}}(t) \mathbf{1}_{i \notin W}\right)\right) \mathrm{d} t \\
& \quad+\sum_{i \in \mathbb{S}} p^{i} \int_{\tau_{0}}^{t_{2}}\left(-\dot{s}^{i}(t)+\frac{\tau-t_{2}}{\tau_{0}-t_{2}}\left(-u^{i}(t)+a^{i} \circ \theta_{\tau_{e}^{j}}(t) \mathbf{1}_{i \notin(W \cup\{j\})}\right)\right) \mathrm{d} t \\
& =\frac{\tau_{e}^{j}-t_{1}}{\tau_{0}-t_{1}} \int_{t_{1}}^{\tau_{0}} H_{W(t)}\left(\theta_{\tau_{e}^{j}}(t), u(t), p\right) \mathrm{d} t+\frac{t_{2}-\tau_{e}^{j}}{t_{2}-\tau_{0}} \int_{\tau_{0}}^{t_{2}} H_{W(t)}\left(\theta_{\tau_{e}^{j}}(t), u(t), p\right) \mathrm{d} t \\
& \quad-\sum_{i \in \mathbb{S}} \int_{t_{1}}^{t_{2}} p^{i} \dot{s}^{i}(t) \mathrm{d} t .
\end{aligned}
$$

Before deriving the Lagrangian, let us introduce some notations. We define the true Hamiltonian by

$$
H_{W}^{*}(t, p)=\min _{v^{i} \in\left[0, \bar{u}^{i}\right]} H_{W}(t, v, p), \quad \forall p \in \mathbb{R}^{n} .
$$

Pontryagin's principle states that

$$
H_{W(t)}^{*}(t, p)=H_{W(t)}(t, u(t), p), \quad \text { for a. a. } t \text { in }[0, T] .
$$

Note that the function $t \mapsto H_{W(t)}^{*}(t, u, p)$ is discontinuous at times $\tau_{e}^{i}$ and $\tau_{b}^{i}$, for all $i$. Indeed, the set of working plants $W(t)$ is changing precisely at these times. The next lemma is a classic useful consequence of Pontryagin's principle. See [4, section 2.4.1, equality (8a)] for a proof.

Lemma 11. Let $u$ be an optimal control, with associated costate $p$. Consider an interval $\left(t_{a}, t_{b}\right)$ included in $[0, T]$ on which none of the plants begins or ends an outage. On such an interval, the costate is constant and the set of working plants is constant, equal to say $W$. The mapping:

$$
h: t \in\left[t_{a}, t_{b}\right] \rightarrow H_{W}^{*}(t, p)
$$


is $C^{1}$ on $\left[t_{a}, t_{b}\right]$ and its derivative is given by

$$
\dot{h}(t)=D_{t} H_{W}^{*}\left(t, p_{0}\right)=D_{t} H_{W}(t, u(t), p), \text { for a. a. } t \text { in }\left[t_{a}, t_{b}\right] .
$$

Proposition 12. The mapping $\tau_{e}^{j} \mapsto \mathcal{L}\left(u, s, p, \tau_{e}^{j}\right)$ is differentiable on $\left(t_{1}, t_{2}\right)$ and

$$
D_{\tau_{e}^{j}} \mathcal{L}\left(u, s, p, \tau_{0}\right)=H_{W}^{*}\left(\tau_{0}, p\right)-H_{W \cup\{j\}}^{*}\left(\tau_{0}, p\right) .
$$

Proof. We have

$$
\begin{aligned}
D_{\tau_{e}^{j}} \mathcal{L}\left(u, s, p, \tau_{e}^{j}\right)= & {\left[\frac{1}{\tau_{0}-t_{1}} \int_{t_{1}}^{\tau_{0}} H_{W}\left(\theta_{\tau_{e}^{j}}(t), u(t), p\right) \mathrm{d} t\right.} \\
& \left.+\frac{\tau_{e}^{j}-t_{1}}{\tau_{0}-t_{1}} \int_{t_{1}}^{\tau_{0}} \frac{t-t_{1}}{\tau_{0}-t_{1}} D_{t} H_{W}\left(\theta_{\tau_{e}^{j}}(t), u(t), p\right) \mathrm{d} t\right] \\
- & {\left[\frac{1}{t_{2}-\tau_{0}} \int_{\tau_{0}}^{t_{2}} H_{W \cup\{j\}}\left(\theta_{\tau_{e}^{j}}(t), u(t), p\right) \mathrm{d} t\right.} \\
& \left.-\frac{t_{2}-\tau_{e}^{j}}{t_{2}-\tau_{0}} \int_{\tau_{0}}^{t_{2}} \frac{t_{2}-t}{t_{2}-\tau_{0}} H_{W \cup\{j\}}\left(\theta_{\tau_{e}^{j}}(t), u(t), p\right) \mathrm{d} t\right] .
\end{aligned}
$$

For $\tau_{e}^{j}=\tau_{0}$, we obtain

$$
\begin{aligned}
& D_{\tau_{e}^{j}} \mathcal{L}\left(u, s, p, \tau_{0}\right) \\
= & \frac{1}{\tau_{0}-t_{1}}\left[\int_{t_{1}}^{\tau_{0}} H_{W}^{*}(t, p) \mathrm{d} t+\int_{t_{1}}^{\tau_{0}}\left(t-t_{1}\right) D_{t} H_{W}^{*}(t, p) \mathrm{d} t\right] \\
& \quad-\frac{1}{t_{2}-\tau_{0}}\left[\int_{\tau_{0}}^{t_{2}} H_{W \cup\{j\}}^{*}(t, p) \mathrm{d} t-\int_{\tau_{0}}^{t_{2}}\left(t_{2}-t\right) D_{t} H_{W \cup\{j\}}^{*}(t, p) \mathrm{d} t\right] .
\end{aligned}
$$

Then, we obtain by integrating by parts (with lemma 11)

$$
\begin{aligned}
& \int_{t_{1}}^{\tau_{0}}\left(t-t_{1}\right) D_{t} H_{W}^{*}(t, p) \mathrm{d} t \\
= & {\left[\left(t-t_{1}\right) H_{W}^{*}(t, p)\right]_{t_{1}}^{\tau_{0}}-\int_{t_{1}}^{\tau_{0}} H_{W}^{*}(t, p) \mathrm{d} t } \\
= & \left(\tau_{0}-t_{1}\right) H_{W}^{*}\left(\tau_{0}, p\right)-\int_{t_{1}}^{\tau_{0}} H_{W}^{*}(t, p) \mathrm{d} t,
\end{aligned}
$$

and a similar expression holds for the integral on $\left[\tau_{0}, t_{2}\right]$. Finally, we obtain

$$
D_{\tau_{e}^{j}} \mathcal{L}\left(u, s, p, \tau_{0}\right)=-\left[H_{W \cup\{j\}}^{*}\left(\tau_{0}, p\right)-H_{W}^{*}\left(\tau_{0}, p\right)\right]
$$

as was to be proved.

Remark 13. In general, there are several solutions to the problem. However, the expression obtained for the derivative of the Lagrangian, when $p$ is given, does not depend on the primal solution, for two reasons:

- the Hamiltonian, and thus the true Hamiltonian, do not depend on the state (and therefore, they do not depend on the past trajectory)

- by definition, the true Hamiltonian at time $t$ does not depend on the choice of the value of the optimal control at time $t$.

$\mathrm{RR} \mathrm{n}^{\circ} 7884$ 
Sensitivity with respect to the beginning of outage The above analysis remains true for $\tau_{b}^{j}$ if hypothesis $(\mathrm{H})$ always holds. In this case, none of the plants (except $j$ ) begins or stops its outage at time $\tau_{b}^{j}$ and we denote by $W$ the set of working plants at the reference time $\tau_{0}(j$ does not belong to $W)$. The only difference with the previous expression is that the $j$-th coordinate of $p$ has a jump at time $\tau_{0}$. Using the conventions (2) and (3), we obtain the expression

$$
D_{\tau_{b}^{j}} \mathcal{L}\left(u, s, p, \tau_{0}\right)=-\left[H_{W}^{*}\left(\tau_{0}, p\left(\tau_{0}^{+}\right)\right)-H_{W \cup\{j\}}^{*}\left(\tau_{0}, p\left(\tau_{0}^{-}\right)\right)\right] .
$$

Notice that the state constraint $s^{j}\left(\tau_{b}^{j}\right)=0$ has become $s^{j}\left(\tau_{0}\right)=0$, as a consequence, it does not depend on $\tau_{b}^{j}$ anymore and we do not need to take it into account in the Lagrangian.

Sensitivity with respect to an arbitrary direction We compute now the value of the directional derivative of the value function in an arbitrary direction. To this purpose, we must realize a complete reparameterization of the problem and some notations are needed. We fix a reference value $\left(\tau_{b, 0}, \tau_{e, 0}\right)$ for the dates of outages and we suppose that hypothesis $(\mathrm{H})$ holds. Then, we can fix dates $t_{b, 1}^{i}, t_{b, 2}^{i}, t_{e, 1}^{i}$ and $t_{e, 2}^{i}$ in $[0, T]$ such that for all $i$ in $\mathbb{S}$,

$$
t_{b, 1}^{i}<\tau_{b, 0}^{i}<t_{b, 2}^{i}<t_{e, 1}^{i}<\tau_{e, 0}^{i}<t_{e, 2}^{i}
$$

and such that on the intervals $\left[t_{b, 1}^{i}, t_{b, 2}^{i}\right]$ and $\left[t_{e, 1}^{i}, t_{e, 2}^{i}\right]$, plant $i$ is the only one to begin or to end its outage. Therefore, we can define the sets $W_{b}^{i}$ and $W_{e}^{i}$ of working plants on the intervals $\left[t_{b, 1}^{i}, t_{b, 2}^{i}\right]$ and $\left[t_{e, 1}^{i}, t_{e, 2}^{i}\right]$ respectively, $i$ being excluded of these sets. The global change of variable to perform is now the following:

$$
\theta_{\tau_{b}, \tau_{e}}\left(t^{\prime}\right)= \begin{cases}t_{b, 1}^{i}+\frac{\tau_{b}^{i}-t_{b, 1}^{i}}{\tau_{b, 0}^{i}-t_{b, 1}^{i}}\left(t^{\prime}-t_{b, 1}^{i}\right), & \text { for } t^{\prime} \text { in }\left[t_{b, 1}^{i}, \tau_{b, 0}^{i}\right], \\ t_{b, 2}^{i}-\frac{t_{b, 2}^{i}-\tau_{b}^{i}}{t_{b, 2}^{i}-\tau_{b, 0}^{i}}\left(t_{b, 2}^{i}-t^{\prime}\right), & \text { for } t^{\prime} \text { in }\left[\tau_{b, 0}^{i}, t_{b, 2}^{i}\right], \\ t_{e, 1}^{i}+\frac{\tau_{e}^{i}-t_{e, 1}^{i}}{\tau_{e, 0}^{i}-t_{e, 1}^{i}}\left(t^{\prime}-t_{e, 1}^{i}\right), & \text { for } t^{\prime} \text { in }\left[t_{e, 1}^{i}, \tau_{e, 0}^{i}\right], \\ t_{e, 2}^{i}-\frac{t_{e, 2}^{i}-\tau_{e}^{i}}{t_{e, 2}^{i}-\tau_{e, 0}^{i}}\left(t_{e, 2}^{i}-t^{\prime}\right), & \text { for } t^{\prime} \text { in }\left[\tau_{e, 0}^{i}, t_{e, 2}^{i}\right], \\ t^{\prime}, & \text { otherwise. }\end{cases}
$$

The general reparameterized problem is the following:

$$
\begin{aligned}
& V\left(\tau_{b}, \tau_{e}\right)= \\
& \min _{u, s}\left[\int_{0}^{T} \dot{\theta}_{\tau_{b}, \tau_{e}}\left(t^{\prime}\right) c\left(d \circ \theta_{\tau_{b}, \tau_{e}}\left(t^{\prime}\right)-\sum_{i \in W\left(t^{\prime}\right)} u^{i}\left(t^{\prime}\right)\right) \mathrm{d} t^{\prime}+\phi(s(T))\right] \\
& \text { s.t. } \forall i \in \mathbb{S} \\
& \quad \dot{s}^{i}\left(t^{\prime}\right)=\dot{\theta}_{\tau_{b}, \tau_{e}}\left(t^{\prime}\right)\left(-u^{i}\left(t^{\prime}\right)+a^{i} \circ \theta_{\tau_{b}, \tau_{e}}\left(t^{\prime}\right) \mathbf{1}_{\left[\tau_{b, 0}^{i}, \tau_{e, 0}^{i}\right]}\left(t^{\prime}\right)\right) \\
& 0 \leq u^{i}\left(t^{\prime}\right) \leq \bar{u}^{i} \\
& s^{i}(0)=s_{0}^{i} \\
& s^{i}\left(\tau_{b, 0}^{i}\right)=0 \\
& s^{i}(T) \geq 0
\end{aligned}
$$

Here, the set of working plants $W\left(t^{\prime}\right)$ at time $t^{\prime}$ is defined by:

$$
W\left(t^{\prime}\right)=\left\{i \in \mathbb{S}, t^{\prime} \notin\left[\tau_{b, 0}^{i}, \tau_{e, 0}^{i}\right]\right\} .
$$


Notations Let us introduce some notations in order to simplify our sensitivity formula. First, we denote by $\Pi\left(\tau_{b, 0}, \tau_{e, 0}\right)$ the set of costates satisfying Pontryagin's principle (lemma 1 ) for the value $\left(\tau_{b, 0}, \tau_{e, 0}\right)$ of the dates of the outages. Recall that it is a subset of $\mathbb{R}^{2 n}$. We also introduce the jumps of the true Hamiltonian, denoted by $\Delta H_{b}^{i}(p)$ and $\Delta H_{e}^{i}(p)$ and defined by

$$
\begin{aligned}
& \Delta H_{b}^{i}(p)=H_{W_{b}^{i}}^{*}\left(\tau_{b, 0}^{i}, p\left(\tau_{b, 0}^{i+}\right)\right)-H_{W_{b}^{i} \cup\{i\}}^{*}\left(\tau_{b, 0}^{i}, p\left(\tau_{b, 0}^{i}\right)\right), \\
& \Delta H_{e}^{i}(p)=H_{W_{e}^{i} \cup\{i\}}^{*}\left(\tau_{e, 0}^{i}, p\left(\tau_{e, 0}^{i}\right)\right)-H_{W_{e}^{i}}^{*}\left(\tau_{e, 0}^{i}, p\left(\tau_{e, 0}^{i}\right)\right),
\end{aligned}
$$

for $p$ in $\Pi\left(\tau_{b, 0}, \tau_{e, 0}\right)$.

Theorem 14. Consider a direction of perturbation denoted by $\left(\delta \tau_{b}, \delta \tau_{e}\right)$. If hypothesis $(\mathrm{H})$ holds, then

$$
\begin{aligned}
& V^{\prime}\left(\left(\tau_{b, 0}, \tau_{e, 0}\right),\left(\delta \tau_{b}, \delta \tau_{e}\right)\right) \\
= & \sup _{p \in \Pi\left(\tau_{b, 0}, \tau_{e, 0}\right)}\left[\sum_{i \in \mathbb{S}}-\delta \tau_{b}^{i} \Delta H_{b}^{i}(p)+\sum_{i \in \mathbb{S}}-\delta \tau_{e}^{i} \Delta H_{e}^{i}(p)\right] .
\end{aligned}
$$

Proof. The expression of the derivative of the Lagrangian given in the thorem is a simple extension of expressions $(22)$ and $(26)$. The theorem is a direct consequence of theorem 8 . For our application problem, a costate is a Lagrange multiplier if and only if it satisfies Pontryagin's principle, since the Hamiltonian is convex. The three hypotheses of the theorem (existence of solutions, qualification and continuity of the derivative of the Lagrangian) are checked in lemmas 17, 18 , and 23 .

\subsection{Study of the Lagrange multipliers}

In this part, we give a complete description of the set $\Pi\left(\tau_{b}, \tau_{e}\right)$ of costates satisfying Pontryagin's principle, which is for our application problem the set of Lagrange multipliers introduced in (13). Note that the characterization of costates holds even if hypothesis $(\mathrm{H})$ is not satisfied.

Notations Let us consider the smallest sequence of times

$$
0=\tau_{0}<\tau_{1}<\cdots<\tau_{M}=T
$$

such that the outages begin or end only at times $\left\{\tau_{1}, \ldots, \tau_{M}\right\}$. For all integer $m$ with $0 \leq m<M$, the set of working plants is constant on the interval of time $\left(\tau_{m}, \tau_{m+1}\right)$.

Let us fix now an optimal control $u$ and its associated trajectory $s$. Since the set of Lagrange multipliers does not depend on the choice of the optimal solution, it suffices to compute the set of costates associated with the particular solution $(u, s)$. We have proved in lemma 6 that $\mu=\sum_{i \in \mathbb{S}} u^{i}(t)$ and $s(T)$ are unique. Let us define, for all $i$ in $\mathbb{S}$,

$$
\begin{cases}\pi_{0}^{i, \min }= & \underset{t \in\left[0, \tau_{b}^{i}\right], u^{i}(t)>0}{\operatorname{ess} \sup _{0}}\left\{-c^{\prime}(d(t)-\mu(t))\right\}, \\ \pi_{0}^{i, \max }= & \underset{t \in\left[0, \tau_{b}^{i}\right], u^{i}(t)<\bar{u}^{i}}{\operatorname{ess} \inf }\left\{-c^{\prime}(d(t)-\mu(t))\right\} .\end{cases}
$$

For all $i$ in $\mathbb{S}$, if $s^{i}(T)>0$, we set

$$
\pi_{T}^{i, \min }=\pi_{T}^{i, \max }=D_{s^{i}} \phi(s(T))
$$


otherwise, we set

$$
\left\{\begin{array}{l}
\pi_{T}^{i, \min }=\underset{t \in\left[\tau_{e}^{i}, T\right], u^{i}(t)>0}{\operatorname{ess} \sup \left\{-c^{\prime}(d(t)-\mu(t))\right\}} \\
\pi_{T}^{i, \max }=\min \left\{\underset{t \in\left[\tau_{e}^{i}, T\right], u^{i}(t)<\bar{u}^{i}}{\operatorname{ess} \inf }\left\{-c^{\prime}(d(t)-\mu(t))\right\}, D_{s^{i}} \phi(s(T))\right\} .
\end{array}\right.
$$

Theorem 15. The set of costates $\Pi\left(\tau_{b}, \tau_{e}\right)$ is described by

$$
\Pi\left(\tau_{b}, \tau_{e}\right)=\left(\prod_{i \in \mathbb{S}}\left[\pi_{0}^{i, \min }, \pi_{0}^{i, \max }\right]\right) \times\left(\prod_{i \in \mathbb{S}}\left[\pi_{T}^{i, \min }, \pi_{T}^{i, \max }\right]\right) .
$$

Proof. Let $t$ in $[0, T]$, let $v$ in $\mathcal{U}$ be such that for all $i \notin W(t), v^{i}=0$. We set $\mu=\sum_{i \in \mathbb{S}} v^{i}$. Fix $q$ in $\mathbb{R}^{n}$. Then $v$ is a solution to the problem of minimization of the Hamiltonian $P_{t, p}$ with $p=q$ if and only if for all $i$ in $W(t)$,

$$
\begin{array}{ll} 
& \left(v^{i}>0 \Rightarrow q^{i}(t) \geq-c^{\prime}(d(t)-\mu)\right), \\
& \left(v^{i}<\bar{u}^{i} \Rightarrow q^{i}(t) \leq-c^{\prime}(d(t)-\mu)\right), \\
\text { and } \quad & i \notin W(t) \Rightarrow q^{i} \leq 0
\end{array}
$$

Therefore, a costate $p$ is such that the Hamiltonian is minimized for almost all $t$ if and only if conditions (33) and (34) are satisfied for almost all $t$ with $q=p(t)$. These conditions being inequalities, it suffices to consider the essential infimum and supremum as we did in the construction of $\pi_{0}^{i, \min }, \pi_{0}^{i, \max }, \pi_{T}^{i, \min }$ and $\pi_{T}^{i, \max }$. Notice that we do not need to impose that $p^{i}(T) \leq 0$, since we already have that $D_{s^{i}} \phi(s(T)) \leq 0$. The theorem follows.

The following lemma describes situations where the costate is unique.

Lemma 16. Let us consider four different cases.

1. (a) If on a non-negligible subset of $\left[0, \tau_{b}^{i}\right], u^{i}(t) \in\left(0, \bar{u}^{i}\right)$, then $p^{i}(0)$ is unique.

(b) If $s^{i}(T)>0$ or if on a non-negligible subset of $\left[\tau_{e}^{i}, T\right], u^{i}(t) \in\left(0, \bar{u}^{i}\right)$, then $p^{i}(T)$ is unique.

2. (a) If there exist two non-negligible subset $\mathcal{T}_{1}$ and $\mathcal{T}_{2}$ of an interval $\left[\tau_{m}, \tau_{m+1}\right]$ with $\tau_{m+1} \leq$ $\tau_{b}^{i}$, such that

$$
\forall t \in \mathcal{T}_{1}, u^{i}(t)=0 \quad \text { and } \quad \forall t \in \mathcal{T}_{2}, u^{i}(t)=\bar{u}^{i},
$$

then, $p^{i}(0)$ is unique.

(b) If the same property holds on an interval $\left[\tau_{m}, \tau_{m+1}\right]$ with $\tau_{m} \geq \tau_{e}^{i}$, then $p^{i}(T)$ is unique.

Proof. In cases 1. $a$ and 1.b, it follows from the existence of a non-negligible subset of $\left[0, \tau_{b}^{i}\right]$ (resp. $\left.\left[\tau_{e}^{i}, T\right]\right)$ where $0<u^{i}(t)<\bar{u}^{i}$ that $\pi_{0}^{i, \min } \geq \pi_{0}^{i, \max }\left(\operatorname{resp} . \pi_{T}^{i, \min } \geq \pi_{T}^{i, \max }\right)$, whence the equality of these bounds and the uniqueness of $p^{i}(0)$ (resp. $p^{i}(T)$ ).

For case 2.a, let us define $r^{\min }$ and $r^{\max }$ by

$$
\begin{aligned}
& r^{\min }=\underset{t \in\left[\tau_{m}, \tau_{m+1}\right], u^{i}(t)>0}{\operatorname{ess} \sup _{t \in\left[\tau_{m}, \tau_{m+1}\right], u^{i}(t)<\bar{u}^{i}}}\left\{-c^{\prime}(d(t)-\mu(t))\right\}, \\
& \left.r^{\max }=c^{\prime}(d(t)-\mu(t))\right\} .
\end{aligned}
$$


Clearly,

$$
-\infty<r^{\min } \leq \pi_{0}^{i, \min } \leq \pi_{0}^{i, \max } \leq r^{\max }<+\infty .
$$

Let us show the uniqueness by contradiction. We suppose that $\pi_{0}^{i, \text { max }}-\pi_{0}^{i, \text { min }}=\varepsilon>0$. It can be observed from lemma 4 that the solution $\mu$ of problem $P_{t, p}^{\prime}$ depends continuously on $d(t)$ on the interval $\left[\tau_{m}, \tau_{m+1}\right]$, since the set of working plants remains constant. The demand $d(t)$ being continuous in time, it follows that $c^{\prime}(d(t)-\mu(t))$ is a continuous function of time. Let $t_{1}$ and $t_{2}$ be two times such that

$$
\begin{aligned}
& -c^{\prime}\left(d\left(t_{1}\right)-\mu\left(t_{1}\right)\right) \leq r^{\min }+\frac{\varepsilon}{3}, \\
& -c^{\prime}\left(d\left(t_{2}\right)-\mu\left(t_{2}\right)\right) \geq r^{\max }-\frac{\varepsilon}{3} .
\end{aligned}
$$

The function $c^{\prime}(d(t)-\mu(t))$ being continuous, there exists a non-negligible subinterval of $\left[t_{1}, t_{2}\right]$ (or $\left[t_{2}, t_{1}\right]$ if $\left.t_{2}<t_{1}\right)$ where $-c^{\prime}(d(t)-\mu(t))$ belongs to $\left[r^{\min }+\varepsilon / 3, r^{\max }-\varepsilon / 3\right]$. On this subinterval, there exists a non-negligible subset where either $0<u^{i}(t)<\bar{u}^{i}$, either $u^{i}(t)=0$ or $u^{i}(t)=\bar{u}^{i}$. In the first case, we obtain the uniqueness of $p^{i}(0)$, which contradicts the statement of nonuniqueness. In the second case, we obtain that

$$
\underset{t \in\left[\tau_{m}, \tau_{m+1}\right], u^{i}(t)<\bar{u}^{i}}{\operatorname{ess} \inf ^{\prime}}\left\{-c^{\prime}(d(t)-\mu(t))\right\} \leq r^{\max }-\frac{\varepsilon}{3},
$$

and in the third case, we obtain that

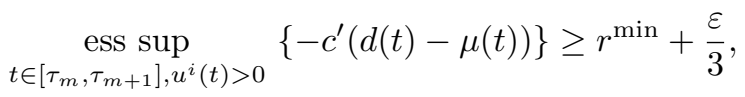

Inequalities (36) and (37) contradict the definition of $r^{\min }$ and $r^{\max }$. Thus, $p^{i}(0)$ is unique. Case 2.b can be treated similarly.

It follows from the contraposition of lemma 16 that if for some $i$ in $\mathbb{S}, p^{i}(0)$ is not unique, then the optimal control $u^{i}$ is constant on each interval $\left(\tau_{m}, \tau_{m+1}\right)$ with $\tau_{m+1} \leq \tau_{b}^{i}$, being equal to 0 or $\bar{u}^{i}$. Denoting by $M_{0}$ the set of indexes $m$ for which $u^{i}(t)=\bar{u}^{i}$ on $\left(\tau_{m}, \tau_{m+1}\right)$, we obtain that

$$
s_{0}^{i}=\bar{u}^{i} \cdot \sum_{m \in M_{0}} \tau_{m+1}-\tau_{m} .
$$

Similarly, if $p^{i}(T)$ is not unique, then $s^{i}(T)=0$ and the optimal control $u^{i}$ is constant on each interval $\left(\tau_{m}, \tau_{m+1}\right)$ with $\tau_{m} \geq \tau_{e}^{i}$, being equal to 0 or $\bar{u}^{i}$. Denoting by $M_{T}$ the set of indexes $m$ for which $u^{i}(t)=\bar{u}^{i}$ on $\left(\tau_{m}, \tau_{m+1}\right)$, we obtain that

$$
\int_{\tau_{b}^{i}}^{\tau_{e}^{i}}=\bar{u}^{i} \cdot \sum_{m \in M_{T}} \tau_{m+1}-\tau_{m} .
$$

Inequalities (38) and 39 are, in some sense, unstable. 


\section{Technical aspects}

In this part, we adopt some new notations in order to simplify the proofs. We set

$$
\ell(t, u)=c\left(d(t)-\sum_{i \in W(t)} u^{i}\right)
$$

and for a sequence of dates $\left(\tau_{b, k}, \tau_{e, k}\right)_{k}$, we denote by $\theta_{k}$ the associated changes of variable, defined by 27) and we obtain, with the new notations:

$$
\begin{aligned}
V\left(\tau_{b, k}, \tau_{e, k}\right)= & \min _{u, s} \int_{0}^{T} \dot{\theta}_{k}(t) \ell\left(\theta_{k}(t), u(t)\right) \mathrm{d} t+\phi(s(T)) \\
\text { s.t. } \forall i \in \mathbb{S}, \quad & \dot{s}^{i}(t)=\dot{\theta}_{k}(t)\left(-u^{i}(t)+a^{i} \circ \theta_{k}(t) \mathbf{1}_{\left[\tau_{b, 0}^{i}, \tau_{e, 0}^{i}\right]}(t)\right), \\
& 0 \leq u^{i}(t) \leq \bar{u}^{i}(t) \\
& s^{i}(0)=s_{0}^{i} \\
& s^{i}\left(\tau_{b, 0}^{i}\right)=0 \\
& s^{i}(T) \geq 0
\end{aligned}
$$

Let us give two elementary properties associated with the changes of variable $\theta_{k}$. First, it can be easily checked that

$$
\theta_{k} \rightarrow \text { Id } \text { and } \dot{\theta}_{k} \rightarrow 1
$$

for the uniform topology of $L^{\infty}\left(0, T ; \mathbb{R}^{n}\right)$. Moreover, if $b$ is in $L^{1}\left(0, T ; \mathbb{R}^{n}\right)$, then

$$
b \circ \theta_{k} \rightarrow b
$$

for the $L^{1}$-topology. This property being easily checked if $b$ is continuous, by density of continuous functions in $L^{1}\left(0, T ; \mathbb{R}^{n}\right)$, we obtain it for all function in $L^{1}\left(0, T ; \mathbb{R}^{n}\right)$.

\subsection{Existence of solutions}

Lemma 17. If condition QC is satisfied, the problem has an optimal solution.

Proof. Consider a minimizing sequence $\left(u_{k}, s_{k}\right)$ of feasible solutions. Since the controls are bounded and the dynamic is linear, one can easily prove with the Banach-Alaoglu theorem and the Arzelà-Ascoli theorem the existence of a subsequence $\left(u_{k}, s_{k}\right)$ such that $u_{k}$ converges to a control $u$ for the weak topology of $L^{\infty}\left(0, T ; \mathbb{R}^{n}\right)$, such that $s_{k}$ converges to a trajectory $s$ for the strong topology of $L^{\infty}\left(0, T ; \mathbb{R}^{n}\right)$, and such that $(u, s)$ is a feasible trajectory. Moreover, the mapping

$$
u \in L^{\infty}\left(0, T ; \mathbb{R}^{n}\right) \mapsto \int_{0}^{T} \ell(t, u(t)) \mathrm{d} t
$$

is sequentially lower semi-continuous for the weak-* topology. Indeed, since for all $t, \ell(t,$.$) is$ differentiable and convex with respect to $u$,

$$
\int_{0}^{T} \ell\left(t, u_{k}(t)\right) \mathrm{d} t \geq \int_{0}^{T} \ell(t, u(t)) \mathrm{d} t+\int_{0}^{T} D_{u} \ell(t, u(t))\left(u_{k}(t)-u(t)\right) \mathrm{d} t,
$$

thus, to the limit,

$$
\int_{0}^{T} \ell(t, u(t)) \mathrm{d} t \leq \liminf _{k \rightarrow \infty} \int_{0}^{T} \ell\left(t, u_{k}(t)\right) \mathrm{d} t .
$$

Since $\phi$ is continuous and the cost function is sequentially weakly-* lower semi-continuous, the trajectory $(u, s)$ is an optimal solution to the problem. 


\subsection{Qualification}

Lemma 18. If condition $\mathrm{QC}$ is satisfied, then Robinson's constraint qualification holds for any feasible trajectory.

Proof. We must check condition ( $\mathrm{RQC}$. We consider that the control $u$ and the trajectory $s$ are the optimization variables, defined on the spaces $L^{\infty}\left(0, T ; \mathbb{R}^{n}\right)$ and $W^{1, \infty}\left(0, T ; \mathbb{R}^{n}\right)$. For simplicity, we denote respectively by $L^{\infty}, L_{+}^{\infty}$ and $W^{1, \infty}$ the spaces $L^{\infty}\left([0, T], \mathbb{R}^{n}\right), L^{\infty}\left(0, T ; \mathbb{R}_{+}^{n}\right)$ and $W^{1, \infty}\left([0, T], \mathbb{R}^{n}\right)$, respectively. The function $G$ describing the constraints is the following:

$$
G:(u, s) \in\left(L^{\infty}, W^{1, \infty}\right) \mapsto\left(G_{E}(u, s), G_{I}(u, s)\right),
$$

where

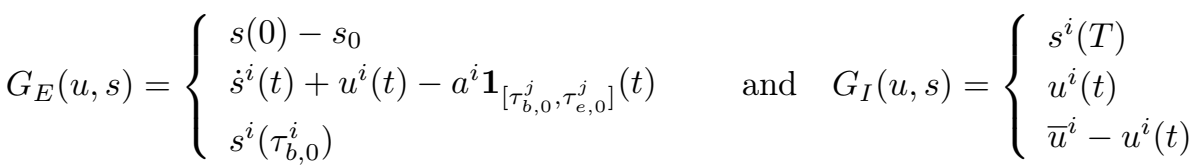

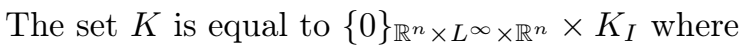

$$
K_{I}=\mathbb{R}_{+}^{n} \times L_{+}^{\infty} \times L_{+}^{\infty} .
$$

Let us consider a feasible trajectory $x=(u, s)$ of the problem, we denote by $d x=(d u, d s)$ a perturbation of the optimization variables $u$ and $s$. We have to characterize the set:

$$
G\left(x, y_{0}\right)+D_{x} G\left(x, y_{0}\right) d x-K \text {. }
$$

An element of this set is of the following form:

$$
\left\{\begin{array}{l}
d s^{i}(0) \\
\dot{d}^{i}(t)+d u^{i}(t) \\
d s^{i}\left(\tau_{b, 0}^{i}\right) \\
s^{i}(T)+d s^{i}(T)-g \\
u^{i}(t)+d u^{i}(t)-\check{u}(t) \\
\bar{u}^{i}-u^{i}(t)-d u^{i}(t)-\hat{u}(t)
\end{array}\right.
$$

where $\check{u}$ and $\hat{u}$ belongs to $L_{+}^{\infty}, g$ belongs to $\mathbb{R}_{+}^{n}$. Note that the expression obtained is decoupled in $i$. This allows us to study the qualification by examining just one coordinate. Let us show that there exists a constant $\varepsilon>0$ such that for all

$$
d g=\left(g_{1}, z, g_{2}, g_{3}, \nu_{1}, \nu_{2}\right) \in \mathbb{R}^{n} \times L^{\infty} \times \mathbb{R}^{n} \times \mathbb{R}^{n} \times L^{\infty} \times L^{\infty}
$$

with $\|d g\|_{\infty} \leq \varepsilon$, there exists $d x=(d u, d s)$ in $L^{\infty} \times W^{1, \infty}$ such that

$$
d g \in G(x, y)+D G_{x}\left(x, y_{0}\right) d x-K .
$$

It is easy to check that this last condition is equivalent to the existence of a control $d u^{i}$ in $L^{\infty}$ satisfying the bounds

$$
\nu_{1}(t) \leq u^{i}(t)+d u^{i}(t) \leq \bar{u}^{i}-\nu_{2}(t),
$$

and such that the associated differential system

$$
\left\{\begin{array}{l}
\dot{d} s^{i}(t)=-d u^{i}(t)+z(t) \\
d s^{i}(0)=g_{1}
\end{array}\right.
$$

$\mathrm{RR} \mathrm{n}^{\circ} 7884$ 
satisfies the following two state constraints:

$$
d s^{i}\left(\tau_{b, 0}^{i}\right)=g_{2}, \quad d s^{i}(T) \geq-s^{i}(T)+g_{3} .
$$

Now, we focus on the construction of $d u^{i}$ on $\left[0, \tau_{b, 0}^{i}\right]$. The idea is to take for $d u^{i}(t)$ a convex combination of its bounds, $\nu_{1}(t)-u^{i}(t)$ and $\bar{u}^{i}-\nu_{2}(t)-u^{i}(t)$. The first state constraint, $d s^{i}\left(\tau_{b, 0}^{i}\right)=$ $g_{2}$ is equivalent to

$$
\int_{0}^{\tau_{b, 0}^{i}} d u^{i}(t)=g_{1}-g_{2}+\int_{0}^{\tau_{b, 0}^{i}} z(t) \mathrm{d} t .
$$

Hypothesis QC states that

$$
0<s_{0}^{i}=\int_{0}^{\tau_{b, 0}^{i}} u^{i}(t) \mathrm{d} t<\tau_{b, 0}^{i} \cdot \bar{u}^{i}
$$

thus we can set

$$
\varepsilon_{1}=\min \left(s_{0}^{i}, \tau_{b, 0}^{i} \cdot \bar{u}^{i}-s_{0}^{i}, \bar{u}^{i}\right)>0 .
$$

We assume that

$$
\left\|\nu_{1}\right\|_{\infty} \leq \frac{\varepsilon_{1}}{2} \min \left(1,\left(\tau_{b, 0}^{i}\right)^{-1}\right) \quad \text { and } \quad\left\|\nu_{2}\right\|_{\infty} \leq \frac{\varepsilon_{1}}{2} \min \left(1,\left(\tau_{b, 0}^{i}\right)^{-1}\right) .
$$

It follows that:

$$
\begin{gathered}
\int_{0}^{\tau_{b, 0}^{i}} \bar{u}^{i}-u^{i}(t)-\nu_{2}(t) \mathrm{d} t \geq\left(\tau_{b, 0}^{i} \bar{u}^{i}-s_{0}^{i}\right)-\tau_{b, 0}^{i} \cdot\left\|\nu_{2}\right\|_{\infty} \geq \varepsilon_{1} / 2, \\
\int_{0}^{\tau_{b, 0}^{i}}-u^{i}(t)-\nu_{1}(t) \mathrm{d} t \leq-s_{0}^{i}+\tau_{b, 0}^{i} \cdot\left\|\nu_{1}\right\|_{\infty} \leq-\varepsilon_{1} / 2,
\end{gathered}
$$

and for all $t$ in $\left[0, \tau_{b, 0}^{i}\right], \nu_{2}(t)-\nu_{1}(t) \leq \bar{u}^{i}$, thus,

$$
-u^{i}(t)-\nu_{1}(t) \leq \bar{u}^{i}-u^{i}(t)-\nu_{2}(t)
$$

We assume that

$$
\left|g_{1}\right| \leq \frac{\varepsilon_{1}}{6}, \quad\left|g_{2}\right| \leq \frac{\varepsilon_{1}}{6}, \quad \text { and } \quad\|z\|_{\infty} \leq \frac{\varepsilon_{1}}{6 \tau_{b, 0}^{i}}
$$

so that

$$
\left|g_{1}-g_{2}+\int_{0}^{\tau_{b, 0}^{i}} z(t) \mathrm{d} t\right| \leq \frac{\varepsilon_{1}}{2}
$$

Let us set

$$
\lambda=\frac{\left(g_{1}-g_{2}+\int_{0}^{\tau_{b, 0}^{i}} z(t) \mathrm{d} t\right)-\left(\int_{0}^{\tau_{b, 0}^{i}}-u^{i}(t)-\nu_{1}(t) \mathrm{d} t\right)}{\int_{0}^{\tau_{b, 0}^{i}} \bar{u}^{i}-\nu_{2}(t) \mathrm{d} t-\int_{0}^{\tau_{b, 0}^{i}}-\nu_{1}(t) \mathrm{d} t},
$$

we obtain, combining (47), 48, and (51) that $0 \leq \lambda \leq 1$. Using 49 and (52), we obtain that the control $d u^{i}$ defined on $\left[0, \tau_{b, 0}^{i}\right]$ by

$$
d u^{i}(t)=\lambda\left[-\nu_{1}(t)-u^{i}(t)\right]+(1-\lambda)\left[\bar{u}^{i}-u^{i}(t)-\nu_{2}(t)\right]
$$

is feasible and that the associated state $d s^{i}(t)$ satisfies the first state constraint. 
Let us focus on the construction of $d u^{i}$ on $\left[\tau_{b, 0}^{i}, T\right]$. The final-state constraint on $d s^{i}(T)$ is satisfied if and only if

$$
\int_{\tau_{b, 0}^{i}}^{T} d u^{i}(t) \leq g_{2}-g_{3}+s^{i}(T)+\int_{\tau_{b, 0}^{i}}^{T} z(t) \mathrm{d} t .
$$

Hypothesis QC states that

$$
0<\int_{\tau_{b, 0}^{i}}^{\tau_{e, 0}^{i}} a^{i}(t) \mathrm{d} t
$$

We set

$$
\varepsilon_{2}=\min \left(\int_{\tau_{b, 0}^{i}}^{\tau_{e, 0}^{i}} a^{i}(t) \mathrm{d} t, \bar{u}^{i}\right)>0 .
$$

We assume now that

$$
\left\|\nu_{1}\right\|_{\infty} \leq \frac{\varepsilon_{2}}{2} \min \left(1,\left(T-\tau_{b, 0}^{i}\right)^{-1}\right) \quad \text { and } \quad\left\|\nu_{2}\right\|_{\infty} \leq \frac{\varepsilon_{2}}{2} .
$$

It follows that:

$$
\begin{aligned}
\int_{\tau_{b, 0}^{i}}^{T}-u^{i}(t)-\nu_{1}(t) \mathrm{d} t & =-\int_{\tau_{b, 0}^{i}}^{\tau_{e, 0}^{i}} a^{i}(t) \mathrm{d} t-\int_{\tau_{b, 0}^{i}}^{T} \nu_{1}(t) \mathrm{d} t+s^{i}(T) \\
& \leq-\varepsilon_{2}+\left(T-\tau_{b, 0}^{i}\right)\left\|\nu_{1}\right\|_{\infty}+s^{i}(T) \\
& \leq-\frac{\varepsilon_{2}}{2}+s^{i}(T)
\end{aligned}
$$

and for all $t$ in $\left[0, \tau_{b, 0}^{i}\right], \nu_{2}(t)-\nu_{1}(t) \leq \bar{u}^{i}$, thus

$$
-u^{i}(t)-\nu_{1}(t) \leq \bar{u}^{i}-u^{i}(t)-\nu_{2}(t) .
$$

Now, we assume that

$$
\left|g_{2}\right| \leq \frac{\varepsilon_{2}}{6}, \quad\left|g_{3}\right| \leq \frac{\varepsilon_{2}}{6}, \quad\|z\|_{\infty} \leq \frac{\varepsilon_{2}}{6\left(T-\tau_{b, 0}^{i}\right)}
$$

so that

$$
g_{2}-g_{3}+\int_{\tau_{b, 0}^{i}}^{T} z(t) \mathrm{d} t \geq-\frac{\varepsilon_{2}}{2} .
$$

Now, we can set, for all $t$ in $\left[\tau_{b, 0}^{i}, T\right]$,

$$
d u^{i}(t)=-u^{i}(t)-\nu_{1}(t)
$$

It follows from 56 that:

$$
\begin{aligned}
d u^{i}(T) & \leq-\varepsilon_{2} / 2+s^{i}(T) \\
& \leq g_{2}-g_{3}+\int_{\tau_{b, 0}^{i}}^{T} z(t) \mathrm{d} t+s^{i}(T) .
\end{aligned}
$$

As a consequence, the second state constraint is satisfied. The lemma is proved by taking for the constant $\varepsilon$ a positive real number satisfying (45), (46), (50), (54), and (55).

$\mathrm{RR} \mathrm{n}^{\circ} 7884$ 


\subsection{On convergence of solutions to the perturbed problems}

The goal of this part is to check the third hypothesis of theorem 8 for our application problem. To that purpose, we fix a reference date $\left(\tau_{b, 0}, \tau_{e, 0}\right)$ and a sequence $\left(\tau_{b, k}, \tau_{e, k}\right)$ of dates converging to $\left(\tau_{b, 0}, \tau_{e, 0}\right)$. We suppose that hypothesis $(\mathrm{H})$ holds for the reference problem. Thus, it holds for $k$ sufficiently large and there exists a sequence of optimal solutions $\left(u_{k}, s_{k}\right)_{k}$ to the perturbed problems (for $k$ sufficiently large). We denote by $\left(p_{k}\right)_{k}$ a sequence of associated costates.

In lemma 19, we obtain the existence of a subsequence of $\left(u_{k}, s_{k}\right)_{k}$ such that $\left(u_{k}\right)_{k}$ converges to an optimal control of the reference problem, $u$, for the weak-* topology, and such that $\left(s_{k}\right)_{k}$ converges uniformly to the associated trajectory $s$. In lemma 21, we prove the existence of a subsequence such that $\left(p_{k}\right)_{k}$ converges to a costate associated with $(u, s)$ and, in lemma 22, we prove that the sum of the controls converges uniformly. Finally, we prove the last hypothesis of theorem 8

Note that all the subsequences have the same name as the original sequence, for the sake of simplicity.

Lemma 19. There exists a subsequence of $\left(u_{k}, s_{k}\right)_{k}$ such that

$$
\begin{aligned}
& u_{k} \stackrel{*}{\rightarrow} u \text { in } L^{\infty}\left(0, T ; \mathbb{R}^{n}\right), \\
& s_{k} \rightarrow s \text { in } L^{\infty}\left(0, T ; \mathbb{R}^{n}\right),
\end{aligned}
$$

where $(u, s)$ is a solution to $\mathcal{P}^{\prime}\left(\tau_{b, 0}, \tau_{e, 0}\right)$.

Proof. In this proof we first show the existence of a feasible limit point $(u, s)$ to the sequence $\left(u_{k}, s_{k}\right)_{k}$. Then, for any feasible trajectory $(\tilde{u}, \tilde{s})$ of the reference problem, we show the existence of a sequence $\left(\tilde{u}_{k}, \tilde{s}_{k}\right)_{k}$ such that both $\left(\tilde{u}_{k}\right)_{k}$ and $\left(\tilde{s}_{k}\right)_{k}$ converges uniformy to $(\tilde{u}, \tilde{s})$ and such that for $k$ sufficiently large, $\left(\tilde{u}_{k}, \tilde{s}_{k}\right)$ is a feasible trajectory for the perturbed problem.

For all $k$, for all $i$ in $\mathbb{S}$ and for all $t$ in $[0, T]$,

$$
\begin{gathered}
\left|\dot{s}_{k}^{i}(t)\right| \leq \bar{u}^{i}+\|a\|_{\infty}, \\
\left|s_{k}^{i}(t)\right| \leq\left|s_{0}^{i}\right|+T\left(\bar{u}^{i}+\|a\|_{\infty}\right),
\end{gathered}
$$

and

$$
\left\|u_{k}^{i}\right\|_{\infty} \leq \bar{u}^{i}
$$

Using the Arzelà-Ascoli theorem and the Banach-Alaoglu theorem, we obtain the existence of a subsequence, still denoted by $\left(u_{k}, s_{k}\right)_{k}$ such that $s_{k}$ converges uniformly to some $s$ in $L^{\infty}\left(0, T ; \mathbb{R}^{n}\right)$, with $s^{i}(0)=s_{0}^{i}, s^{i}\left(\tau_{b, 0}^{i}\right)=0$ and $s^{i}(T) \geq 0$ and such that $u_{k}$ converges to some $u$ for the weak-* topology of $L^{\infty}\left(0, T ; \mathbb{R}^{n}\right)$. Necessarily, for almost all $t$ in $[0, T], 0 \leq u^{i}(t) \leq \bar{u}^{i}$ and, for all $k$ and for all $t^{\prime}$,

$$
\begin{aligned}
s_{k}^{i}\left(t^{\prime}\right)=s_{0}^{i} & +\int_{0}^{t^{\prime}} \dot{\theta}_{k}(t)\left(-u_{k}^{i}(t)+a^{i} \circ \theta_{k}(t) \mathbf{1}_{\left[\tau_{b, 0}^{i}, \tau_{e, 0}^{i}\right]}(t)\right) \mathrm{d} t \\
=s_{0}^{i} & +\int_{0}^{t^{\prime}}-\left(\dot{\theta}_{k}(t)-1\right) u_{k}^{i}(t) \mathrm{d} t+\int_{0}^{t^{\prime}}\left(\dot{\theta}_{k}(t)-1\right) a^{i} \circ \theta_{k}(t) \mathbf{1}_{\left[\tau_{b, 0}^{i}, \tau_{e, 0}^{i}\right]}(t) \mathrm{d} t \\
& +\int_{0}^{t^{\prime}}-\left(u_{k}^{i}(t)-u^{i}(t)\right) \mathrm{d} t+\int_{0}^{t^{\prime}}\left(a^{i} \circ \theta_{k}(t)-a^{i}(t)\right) \mathbf{1}_{\left[\tau_{b, 0}^{i}, \tau_{e, 0}^{i}\right]}(t) \mathrm{d} t \\
& +\int_{0}^{t^{\prime}}-u^{i}(t)+a^{i}(t) \mathbf{1}_{\left[\tau_{b, 0}^{i}, \tau_{e, 0}^{i}\right]}(t) \mathrm{d} t .
\end{aligned}
$$


Using 40, 41, and the weak-* convergence of $\left(u_{k}\right)_{k}$, we obtain, to the limit,

$$
s^{i}(t)=s_{0}^{i}+\int_{0}^{t^{\prime}}-u^{i}(t)+a^{i}(t) \mathbf{1}_{\left[\tau_{b, 0}^{i}, \tau_{e, 0}^{i}\right]}(t) \mathrm{d} t
$$

which proves that $s$ satisfies the differential equation of the reference problem, hence $(u, s)$ is feasible.

Let $(\tilde{u}, \tilde{s})$ be a feasible control of the reference problem. It can be proved (with the same kind of estimates as in (57) that

$$
\begin{aligned}
& s_{0}^{i}+\int_{0}^{\tau_{b, 0}^{i}} \dot{\theta}_{k}(t)\left(-\tilde{u}^{i}(t)+a^{i} \circ \theta_{k}(t) \mathbf{1}_{\left[\tau_{b, 0}^{i}, \tau_{e, 0}^{i}\right]}(t)\right) \mathrm{d} t=\tilde{s}\left(\tau_{b, 0}^{i}\right)+o(1)=o(1), \\
& s_{0}^{i}+\int_{0}^{T} \dot{\theta}_{k}(t)\left(-\tilde{u}^{i}(t)+a^{i} \circ \theta_{k}(t) \mathbf{1}_{\left[\tau_{b, 0}^{i}, \tau_{e, 0}^{i}\right]}(t)\right) \mathrm{d} t=\tilde{s}(T)+o(1)=o(1),
\end{aligned}
$$

Since Robinson's qualification holds for the trajectory $(\tilde{u}, \tilde{s})$ by lemma 18 , we obtain, using the stability theorem 2.87 in [1] that there exists a sequence of feasible trajectories $\left(\tilde{u}_{k}, \tilde{s}_{k}\right)$ for the perturbed problems such that $\left(\tilde{u}_{k}\right)_{k}$ and $\left(\tilde{s}_{k}\right)_{k}$ converges uniformly to $\tilde{u}$ and $\tilde{s}$ respectively.

Finally, we have that

$$
\int_{0}^{T} \ell\left(t, u_{k}(t)\right) \mathrm{d} t \leq \int_{0}^{T} \ell\left(t, \tilde{u}_{k}(t)\right) \mathrm{d} t
$$

thus passing to the lim inf in the left-hand-side (like in 43) and passing to the limit in the right-hand-side, we obtain that

$$
\int_{0}^{T} \ell(t, u(t)) \mathrm{d} t \leq \int_{0}^{T} \ell(t, \tilde{u}(t)) \mathrm{d} t,
$$

which proves the optimality of $(u, s)$. The lemma follows.

Lemma 20. The sequence $\left(p_{k}\right)$ is bounded.

Proof. This result derives from the study of $\Pi\left(\tau_{b, 0}, \tau_{e, 0}\right)$ conducted in theorem 15. The qualification condition (QC) being stable, it is satisfied for $k$ sufficiently large. When the qualification condition is satisfied, it is impossible that $u^{i}(t)=0$ for almost all $t$ in $\left[0, \tau_{b, 0}^{i}\right]$ or that $u^{i}(t)=\bar{u}^{i}$ for almost all $t$ in $\left[0, \tau_{b, 0}^{i}\right]$, thus the associated bounds $\pi_{0}^{i, \min }$ and $\pi_{0}^{i, \max }$ are finite. More precisely, denoting respectively by $d_{\min }$ and $d_{\max }$ the infimum and the supremum of $d$ over $[0, T]$, we obtain that

$$
-c^{\prime}\left(d_{\max }\right) \leq p_{k}^{i}(0) \leq-c^{\prime}\left(d_{\min }-\sum_{i \in \mathbb{S}} \bar{u}^{i}\right),
$$

since $-c^{\prime}$ is non-increasing. This proves the boundedness of $p_{k}(0)$. For the study of $p_{k}(T)$, let us recall first that up to a subsequence, a sequence $\left(u_{k}, s_{k}\right)$ of optimal solutions to the perturbed problems is such that $s_{k}(T)$ converges. Let $B_{T}$ a compact of $\mathbb{R}^{n}$ be such that $s_{k}(T)$ belongs to $B_{T}$ for $k$ big enough. There are two cases: if $s_{k}^{i}(T)>0$, then

$$
\inf _{s \in B_{T}} D_{s^{i}} \phi(s) \leq D_{s^{i}} \phi\left(s_{k}(T)\right)=p_{k}^{i}(T) \leq \sup _{s \in B_{T}} D_{s^{i}} \phi(s)
$$

otherwise, $s_{k}^{i}(T)=0$ and by qualification, it is impossible to have $u_{k}^{i}(t)=0$ for all $t$ in $\left[\tau_{e, 0}^{i}, T\right]$ in this case, thus

$$
-c^{\prime}\left(d_{\max }\right) \leq p_{k}^{i}(T) \leq \sup _{s \in B_{T}} D_{s^{i}} \phi(s) .
$$

$\mathrm{RR} \mathrm{n}^{\circ} 7884$ 
Finally, we obtain that

$$
\min \left\{-c^{\prime}\left(d_{\max }\right), \inf _{s \in B_{T}} D_{s^{i}} \phi(s)\right\} \leq p_{k}^{i}(T) \leq \sup _{s \in B_{T}} D_{s^{i}} \phi(s),
$$

whence the boundedness of $\left(p_{k}\right)_{k}$.

Lemma 21. Up to a subsequence, $\left(p_{k}\right)_{k}$ converges to some $p$ in $\Pi\left(\tau_{b, 0}, \tau_{e, 0}\right)$.

Proof. Recall that $p$ is viewed as an element of $\mathbb{R}^{2 n}$, therefore we do not need to be precise about the topology involved for the convergence. By lemma 19, we can extract from this sequence a sequence of solutions, denoted by $\left(u_{k}, s_{k}\right)$ such that $u_{k} \stackrel{*}{\rightarrow} u$ and $s_{k} \rightarrow s\left(\right.$ in $\left.L^{\infty}\left(0, T ; \mathbb{R}^{n}\right)\right)$ where $(u, s)$ is a solution to $\mathcal{P}\left(\tau_{b, 0}, \tau_{e, 0}\right)$.

By lemma 20, the sequences $p_{k}(0)$ and $p_{k}(T)$ are bounded, and thus we can extract a subsequence such that these sequences converge to say $p_{0}$ and $p_{T}$. Let us prove that $p=\left(p_{0}, p_{T}\right)$ belongs to $\Pi\left(\tau_{b, 0}, \tau_{e, 0}\right)$. Recall that the Hamiltonian associated the perturbed problem is

$$
\dot{\theta}_{k}(t) H_{W(t)}\left(\theta_{k}(t), u, p\right) .
$$

Let $a$ and $b$ be such that $0 \leq a<b \leq T$, let $v$ in $L^{\infty}(0, T)$ be such that for almost all $t$ in $[0, T]$, for all $i$ in $\mathbb{S}, 0 \leq v^{i}(t) \leq \bar{u}^{i}$. In order to show that $p$ belongs to $\Pi\left(\tau_{b, 0}, \tau_{e, 0}\right)$, it suffices to show that:

$$
\int_{a}^{b} H_{W(t)}(t, p(t), u(t)) \mathrm{d} t \leq \int_{a}^{b} H_{W(t)}(t, p(t), v(t)) \mathrm{d} t .
$$

Applying Pontryagin's principle to the perturbed problem, we obtain directly that

$$
\int_{a}^{b} H_{W(t)}\left(\theta_{k}(t), u_{k}(t), p_{k}(t)\right) \mathrm{d} t \leq \int_{a}^{b} H_{W(t)}\left(\theta_{k}(t), v(t), p_{k}(t)\right) \mathrm{d} t .
$$

Let us focus on the integral of the left-hand-side. We have

$$
\begin{aligned}
& \int_{a}^{b} H_{W(t)}\left(\theta_{k}(t), u_{k}(t), p_{k}(t)\right) \mathrm{d} t \\
= & \int_{a}^{b} H_{W(t)}\left(\theta_{k}(t), u_{k}(t), p_{k}(t)\right)-H_{W(t)}\left(t, u_{k}(t), p_{k}(t)\right) \mathrm{d} t \\
& +\int_{a}^{b} H_{W(t)}\left(t, u_{k}(t), p_{k}(t)\right)-H_{W(t)}\left(t, u_{k}(t), p(t)\right) \mathrm{d} t \\
& +\int_{a}^{b} H_{W(t)}\left(t, u_{k}(t), p(t)\right)-H_{W(t)}(t, u(t), p(t)) \mathrm{d} t \\
& +\int_{a}^{b} H_{W(t)}(t, u(t), p(t)) \mathrm{d} t .
\end{aligned}
$$

Using (40), (41), the uniform convergence of $\left(p_{k}\right)_{k}$, the weak-* lower semi-continuity of the integral of the Hamiltonian (see (43) for the idea of a proof), we obtain that to the limit,

$$
\liminf _{k \rightarrow \infty} \int_{a}^{b} \theta_{k}^{\prime}(t) H_{W(t)}\left(\theta_{k}(t), u_{k}(t), p_{k}(t)\right) \mathrm{d} t \leq \int_{a}^{b} H_{W(t)}(t, u(t), p(t)) \mathrm{d} t .
$$

Similarly, we can show that

$$
\lim _{k \rightarrow \infty} \int_{a}^{b} \theta_{k}^{\prime}(t) H_{W(t)}\left(\theta_{k}(t), v(t), p_{k}(t)\right) \mathrm{d} t=\int_{a}^{b} H_{W(t)}(t, v(t), p(t)) \mathrm{d} t .
$$


Thus, passing to the limit in 58 , we obtain

$$
\int_{a}^{b} H_{W(t)}(t, u(t), p(t)) \mathrm{d} t \leq \int_{a}^{b} H_{W(t)}(t, v(t), p(t)) \mathrm{d} t,
$$

which proves that $p$ belongs to $\Pi\left(\tau_{b, 0}, \tau_{e, 0}\right)$, hence the lemma.

Lemma 22. Up to a subsequence,

$$
\sum_{i \in \mathbb{S}} u_{k}^{i} \longrightarrow \sum_{i \in \mathbb{S}} u^{i} \text { in } L^{\infty}\left(0, T ; \mathbb{R}^{n}\right) .
$$

Proof. As usual, we set $\mu_{k}(t)=\sum_{i \in \mathbb{S}} u_{k}^{i}(t)$. Let us set

$$
\begin{aligned}
\tilde{H}_{W}: \mathbb{R} \times \mathbb{R}^{n} \times \mathbb{R}^{n} & \rightarrow \mathbb{R} \\
(d, \mu, p) & \mapsto c(d(t)-\mu)+\xi_{W}(\mu, p),
\end{aligned}
$$

where $W$ is a given subset of $\mathbb{S}$. This function looks like a Hamiltonian, however, notice that the demand is viewed as a parameter now. Moreover, the part involving the refuelling $a(t)$ is missing. For almost all $t$ in $[0, T]$,

$$
\mu_{k}(t)=\min _{\mu \in\left[0, \sum_{i \in W(t)} \bar{u}^{i}\right]} \tilde{H}_{W(t)}\left(d \circ \theta_{k}(t), \mu, p_{k}(t)\right) .
$$

Thanks to the reparameterization, when $t$ is given, $\mu_{k}(t)$ minimizes a function $\tilde{H}_{W(t)}$ independent on $k$. Recall that the cost function $c$ is $\alpha$-convex and so is the function $\mu \mapsto \tilde{H}_{W}(d, p, \mu)$. Considering that the optimization problem given by $\sqrt[59]{ }$ is a problem parameterized by $d \circ \theta_{k}$ and $p_{k}$, we obtain by a classical property of stability of optimal solutions (see [1, proposition 4.32]) that there exists a constant $A$ independent on time such that for almost all $t$ in $[0, T]$,

$$
\left|\mu_{k}(t)-\mu(t)\right| \leq A\left(\left|p_{k}(t)-p(t)\right|+\left|d \circ \theta_{k}(t)-d(t)\right|\right),
$$

By lemma 21, we know that up to a subsequence, $\left(p_{k}(0), p_{k}(T)\right)$ converges to some $p$ in $\Pi\left(\tau_{b}, \tau_{e}\right)$. Since the times of discontinuity of $p$ are fixed, this implies the uniform convergence of the costate, when considered as a time function. Moreover, it is easy to check that the sequence $\left(d \circ \theta_{k}(t)\right)_{k}$ converges uniformly to $d(t)$, since $d(t)$ is Lipschitz and since $\left(\theta_{k}\right)_{k}$ converges uniformly to the identity function on $[0, T]$. Together with $[60)$, we obtain that

$$
\left\|\mu_{k}-\mu\right\|_{\infty} \leq A\left(\left\|p_{k}-p\right\|_{\infty}+\left\|d \circ \theta_{k}-d\right\|_{\infty}\right) \rightarrow 0 .
$$

as was to be proved.

Lemma 23. If hypotheses $(\mathrm{H})$ and $(\mathrm{QC})$ hold, then hypothesis 3 of theorem 8 is satisfied for any direction of perturbation.

Proof. We know by lemmas 19 and 22 that up to a subsequence, for all $i$ in $\mathbb{S}$,

$$
u_{k}^{i} \stackrel{*}{\rightarrow} u^{i} \quad \text { and } \quad \mu_{k}=\sum_{i \in \mathbb{S}} u_{k}^{i} \rightarrow \mu=\sum_{i \in \mathbb{S}} u^{i} .
$$

Consider a sequence of times $\left(\tilde{\tau}_{b, k}, \tilde{\tau}_{e, k}\right)$ such that for all $k$,

$$
\left(\tilde{\tau}_{b, k}, \tilde{\tau}_{e, k}\right) \in\left[\left(\tau_{b, 0}, \tau_{e, 0}\right),\left(\tau_{b, k}, \tau_{e, k}\right)\right] .
$$

$\mathrm{RR} \mathrm{n}^{\circ} 7884$ 
For simplicity, we consider that the direction of perturbation is a unit basic vector in direction $\tau_{e}^{j}$, so that we can refer to expression (23) for the derivative of the Lagrangian, and we use the same notations. We have:

$$
\begin{aligned}
D_{\tau_{e}^{j}} \mathcal{L}\left(u_{k}, s, p,\left(\tilde{\tau}_{b, k}, \tilde{\tau}_{e, k}\right)\right) & {\left[\frac{1}{\tau_{0}-t_{1}} \int_{t_{1}}^{\tau_{0}} H_{W}\left(\theta_{\tilde{\tau}_{b, k}, \tilde{\tau}_{e, k}}(t), u_{k}(t), p\right) \mathrm{d} t\right.} \\
= & \left.\quad+\frac{\tilde{\tau}_{e, k}^{j}-t_{1}}{\tau_{0}-t_{1}} \int_{t_{1}}^{\tau_{0}} \frac{t-t_{1}}{\tau_{0}-t_{1}} D_{t} H_{W}\left(\theta_{\tilde{\tau}_{b, k}, \tilde{\tau}_{e, k}}(t), u_{k}(t), p\right) \mathrm{d} t\right] \\
& -\left[\frac{1}{t_{2}-\tau_{0}} \int_{\tau_{0}}^{t_{2}} H_{W \cup\{j\}}\left(\theta_{\tilde{\tau}_{b, k}, \tilde{\tau}_{e, k}}(t), u_{k}(t), p\right) \mathrm{d} t\right. \\
& \left.\quad-\frac{t_{2}-\tilde{\tau}_{e, k}^{j}}{t_{2}-\tau_{0}} \int_{\tau_{0}}^{t_{2}} \frac{t_{2}-t}{t_{2}-\tau_{0}} D_{t} H_{W \cup\{j\}}\left(\theta_{\tilde{\tau}_{b, k}, \tilde{\tau}_{e, k}}(t), u_{k}(t), p\right) \mathrm{d} t\right] .
\end{aligned}
$$

Moreover, for all $t$ in $\left[t_{1}, \tau_{0}\right]$,

$$
\begin{aligned}
& H_{W}\left(\theta_{\tilde{\tau}_{b, k}, \tilde{\tau}_{e, k}}(t), u_{k}(t), p\right) \\
= & c\left(d \circ \theta_{\tilde{\tau}_{b, k}, \tilde{\tau}_{e, k}}(t)-\sum_{i \in W} u_{k}(t)\right)+\sum_{i \in \mathbb{S}} p^{i}\left(-u^{i}(t)+a^{i} \circ \theta_{\tilde{\tau}_{b, k}, \tilde{\tau}_{e, k}}(t) \mathbf{1}_{i \notin W}\right),
\end{aligned}
$$

thus, using the strong convergence of the sum of controls, the weak-* convergence of controls, the strong convergence of $\theta_{\tilde{\tau}_{b, k}, \tilde{\tau}_{e, k}}$ and 441 , we obtain that

$$
\int_{0}^{T} H_{W}\left(\theta_{\tilde{\tau}_{b, k}, \tilde{\tau}_{e, k}}(t), u_{k}(t), p\right) \mathrm{d} t \rightarrow \int_{0}^{T} H_{W}(t, u(t), p) \mathrm{d} t
$$

and we prove similarly the convergence of the integral of $D_{t} H_{W}$, and finally, we obtain that for a subsequence of the original sequence $\left(\tilde{\tau}_{b, k}, \tilde{\tau}_{e, k}\right)_{k}$,

$$
D_{\tau_{e}^{j}} \mathcal{L}\left(u_{k}, s, p,\left(\tilde{\tau}_{b, k}, \tilde{\tau}_{e, k}\right)\right) \rightarrow D_{\tau_{e}^{j}} \mathcal{L}\left(u, s, p,\left(\tau_{b, 0}, \tau_{e, 0}\right)\right) .
$$

This property easily extends to all direction of perturbation by linearity of the derivative of the Lagrangian. 


\section{References}

[1] J. Frédéric Bonnans and Alexander Shapiro. Perturbation analysis of optimization problems. Springer Series in Operations Research. Springer-Verlag, New York, 2000.

[2] Fabrice Fourcade, Ellis Johnson, Mourad Bara, and Philippe Cortey-Dumont. Optimizing nuclear power plant refueling with mixed-integer programming. European Journal of Operational Research, 97(2):269 - 280, 1997.

[3] E. G. Gol'šteĭn. Theory of convex programming. American Mathematical Society, Providence, R.I., 1972. Translated from the Russian by K. Makowski, Translations of Mathematical Monographs, Vol. 36.

[4] A. D. Ioffe and V. M. Tihomirov. Theory of extremal problems, volume 6 of Studies in Mathematics and its Applications. North-Holland Publishing Co., Amsterdam, 1979. Translated from the Russian by Karol Makowski.

[5] Mohand Khemmoudj, Marc Porcheron, and Hachemi Bennaceur. When constraint programming and local search solve the scheduling problem of Electricite de France nuclear power plant outages. In Frédéric Benhamou, editor, Principles and Practice of Constraint Programming - CP 2006, volume 4204 of Lecture Notes in Computer Science, pages 271-283. Springer Berlin / Heidelberg, 2006.

[6] Mohand Ou Idir Khemmoudj. Modélisation et résolution de systèmes de contraintes : Application au problème de placement des arrêts et de la production des réacteurs nucléaires d'EDF. PhD Thesis, Avril 2007.

[7] Marc Porcheron, Agnès Gorge, Olivier Juan, Thomas Simovic, and Guillaume Dereu. Challenge ROADEF/EURO 2010: A large-scale energy management problem with varied constraints. http://challenge.roadef.org/2010/files/sujetEDFv22.pdf. Février 2010. 


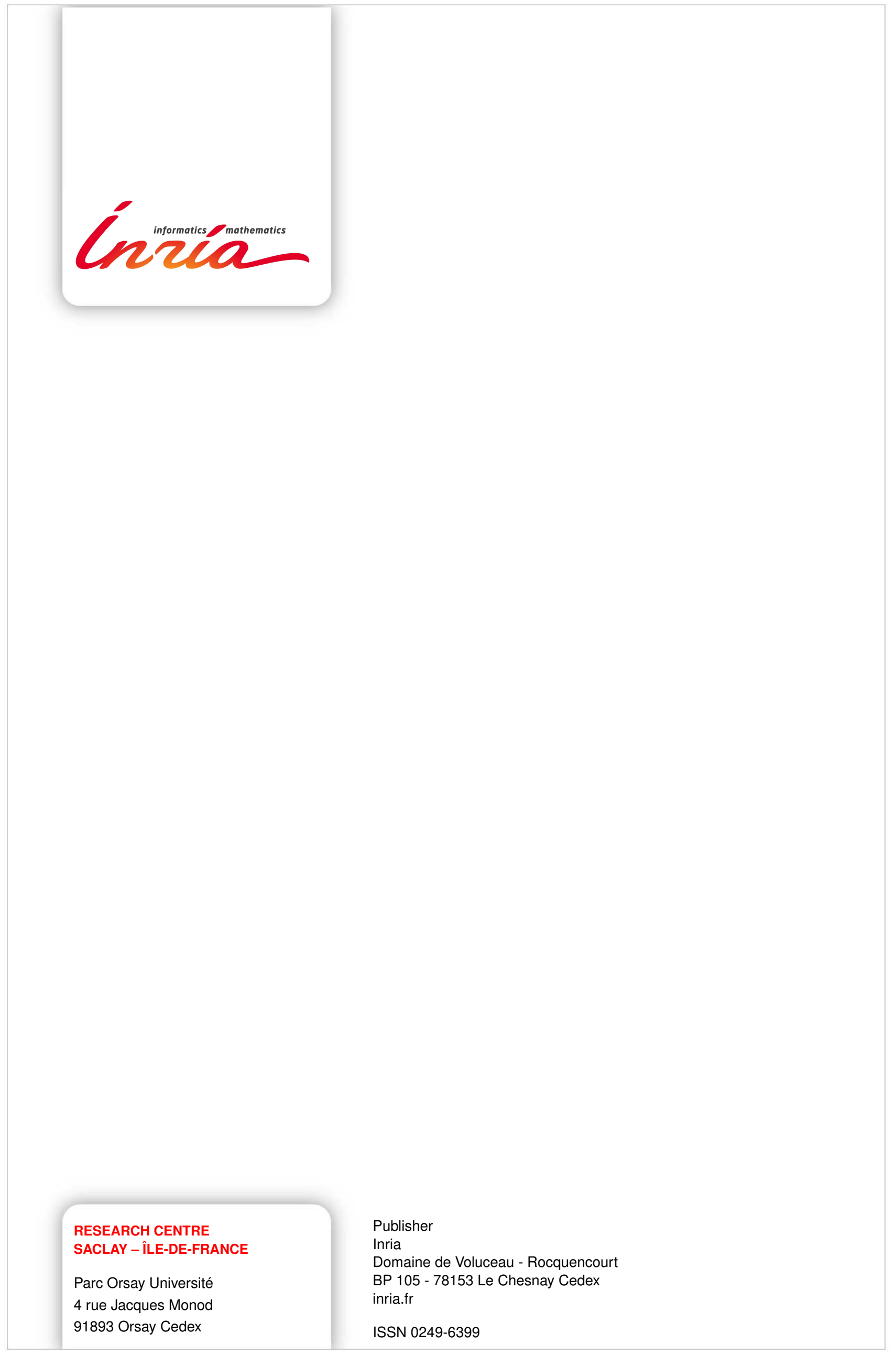

